\title{
CREMONA LINEARIZATIONS OF SOME CLASSICAL VARIETIES
}

\author{
CIRO CILIBERTO, MARIA ANGELICA CUETO, MASSIMILIANO MELLA, KRISTIAN RANESTAD, \\ AND PIOTR ZWIERNIK
}

\begin{abstract}
In this paper we present an effective method for linearizing rational varieties of codimension at least two under Cremona transformations, starting from a given parametrization. Using these linearizing Cremonas, we simplify the equations of secant and tangential varieties of some classical examples, including Veronese, Segre and Grassmann varieties. We end the paper by treating the special case of the Segre embedding of the $n$-fold product of projective spaces, where cumulant Cremonas, arising from algebraic statistics, appear as specific cases of our general construction.
\end{abstract}

\section{INTRODUCTION}

Computations in Algebraic Geometry may be very sensitive to the choice of coordinates. Often, by picking the appropriate coordinate system, calculations or expressions can be greatly simplified. Change of variables with rational functions are classically known as Cremona transformations and give a huge flexibility when dealing with systems of polynomial equations. In this paper, we focus on varieties and maps defined over $\mathbb{C}$.

Cremona transformations, one of the most venerable topics in algebraic geometry, were widely studied in the second half of XIX and the first of XX century. They became again fashionable in recent times, after the spectacular developments of birational geometry due to Mori, Kawamata, Kollár, et al. and even more recently by Birkar, Cascini, Hacon, McKernan (see [7] and references therein). However, despite this great progress, Cremona transformations still reserve a great deal of surprises. The aim of the present, mainly expository, paper is to show how useful they can be in studying some classical geometric invariants of complex projective varieties, linking previous independent work of the last three authors (see $[9,12,17])$. For a recent survey on the properties of the group of Cremona transformations, we refer the reader to [3].

The work of Mella and Polastri [12] shows that any rational variety $X$ of codimension at least two in $\mathbb{P}^{r}$ can be linearized by a Cremona transformation: a linearizing Cremona map is a transformation that maps $X$ birationally to a linear subspace of $\mathbb{P}^{r}$. In Section 2 we provide a proof of the aforementioned result, close to the original one in spirit, but best suited for effective computations.

If $X$ in $\mathbb{P}^{r}$ admits a birational linear projection to a linear subspace, a Cremona linearizing map can be directly constructed as a triangular Cremona transformation (see $\S 1.4$ ). In fact, in Section 1 we present a systematic approach to Cremona transformations linearizing a rational variety. They turn out to be building blocks for the cumulant Cremona transformations, which we present in Section 5. In Section 3 we discuss the effect of linearizing Cremona transformations on tangential and secant varieties. We devote Section 4 to the study of a number of classical examples, including Veronese, Segre and Grassmann varieties, and in these cases we observe an interesting feature of linearizing Cremonas: they tend to simplify also tangential and secant varieties.

The final Section 5 is, as we said, devoted to cumulant Cremona transformations which appear already, in a simple case, in Section 4. Cumulants arise from algebraic statistics (see [17]) and can be viewed as the choice of preferable coordinates in which varieties coming from algebraic statistics simplify. For instance, Sturmfels and Zwiernik [16] used cumulants to simplify the equations of the Segre embedding of the $n$-fold product of $\mathbb{P}^{1}$ and of its tangent variety. More recent results in the same direction are contained in $[11,13]$. Cumulants are particular instances of linearizing Cremona transformations. We conclude by indicating how to generalize some combinatorial formulas in $[13,16,17]$.

Date: November 5, 2018

2010 Mathematics Subject Classification. Primary 14E25 ; Secondary 14E08, 14N05, 14E05.

Key words and phrases. Cremona transformations, Birational maps, Segre and Veronese varieties, Cumulants, Secant cumulants. 


\section{Construction of some Cremona transformations}

In this section we present some recipes for constructing Cremona transformations. We focus on two specific closely-related types: monoidal extensions and triangular Cremona transformations. All constructions in this paper may be seen as iterated applications of monoidal extensions as in Section 1.3 (see also [4]).

\subsection{Basics on Cremona transformations. A Cremona transformation is a birational map}

$$
\varphi: \mathbb{P}^{r} \rightarrow \mathbb{P}^{r}, \quad\left[x_{0}, \ldots, x_{r}\right] \mapsto\left[F_{0}\left(x_{0}, \ldots, x_{r}\right), \ldots, F_{r}\left(x_{0}, \ldots, x_{r}\right)\right],
$$

where $F_{i}\left(x_{0}, \ldots, x_{r}\right)$ are coprime homogeneous polynomials of the same degree $\delta>0$, for $0 \leqslant i \leqslant r$. The inverse map is also a Cremona transformation, and it is defined by coprime homogeneous polynomials $G_{i}\left(x_{0}, \ldots, x_{r}\right)$ of degree $\delta^{\prime}>0$, for $0 \leqslant i \leqslant r$. In this case, we say that $\varphi$ is a $\left(\delta, \delta^{\prime}\right)$-Cremona transformation. The subscheme $\operatorname{Ind}(\varphi):=\left\{F_{i}\left(x_{0}, \ldots, x_{r}\right)=0\right\}_{0 \leqslant i \leqslant r}$ is the indeterminacy locus of $\varphi$. Since the composition of $\varphi$ and its inverse is the identity, we have

$$
G_{i}\left(F_{0}\left(x_{0}, \ldots, x_{r}\right), \ldots, F_{r}\left(x_{0}, \ldots, x_{r}\right)\right)=\Phi \cdot x_{i}, \text { for } 0 \leqslant i \leqslant r
$$

where $\Phi$ is a homogeneous polynomial of degree $\delta \cdot \delta^{\prime}-1$. The hypersurface Fund $(\varphi):=\{\Phi=0\}$ is the fundamental locus of $\varphi$ and its support is the reduced fundamental locus $\operatorname{Fund}_{\text {red }}(\varphi)$. In general one cannot reconstruct $\operatorname{Fund}(\varphi)$ from $\operatorname{Fund}_{\text {red }}(\varphi)$, except when $\operatorname{Fund}_{\text {red }}(\varphi)$ is irreducible. Indeed, in this case, we recover Fund $(\varphi)$ from its multiplicity value $\left(\delta \cdot \delta^{\prime}-1\right) / e$, where $e$ is the degree of $\operatorname{Fund}_{\text {red }}(\varphi)$. By construction, $\operatorname{Ind}(\varphi) \subset \operatorname{Fund}(\varphi)$ and $\phi$ is one-to-one on the complement of $\operatorname{Fund}_{\text {red }}(\varphi)$.

Often times in this paper, the $\operatorname{loci}_{\operatorname{Fund}}(\varphi)$ and $\operatorname{Fund}_{\text {red }}\left(\varphi^{-1}\right)$ induced by the Cremona transformation $\varphi$ are the same hyperplane. In those cases, we can see $\varphi$ as a polynomial automorphism $\varphi_{a}: \mathbb{A}^{r} \rightarrow \mathbb{A}^{r}$ (often denoted by $\varphi$ ) whose extension to $\mathbb{P}^{r}$ contains the hyperplane at infinity as its fundamental locus.

1.2. Monoids. Let $X \subset \mathbb{P}^{r}$ be a hypersurface of degree $d$ where $r \geqslant 2$. We say that $X$ is a monoid with vertex $p \in \mathbb{P}^{r}$ if $X$ is irreducible and $p$ is a point in $X$ of multiplicity exactly $d-1$. Note that a monoid can have more than one vertex. If we choose projective coordinates in such a way that $p=[0, \ldots, 0,1]$, then the defining equation of $X$ is

$$
f\left(x_{0}, \ldots, x_{r}\right)=f_{d-1}\left(x_{0}, \ldots, x_{r-1}\right) x_{r}+f_{d}\left(x_{0}, \ldots, x_{r-1}\right)=0,
$$

where $f_{d-1}$ and $f_{d}$ are homogeneous polynomials of degree $d-1$ and $d$ respectively and $f_{d-1}$ is nonzero. The hypersurface $X$ is irreducible if and only if $f_{d-1}$ and $f_{d}$ are coprime.

A monoid $X$ is rational. Indeed, the projection of $X$ from its vertex $p$ onto a hyperplane $H$ not passing through $p$ is a birational map $\pi: X \rightarrow H \cong \mathbb{P}^{r-1}$. If $H$ has equation $x_{r}=0$, then the inverse map $\pi^{-1}: \mathbb{P}^{r-1} \rightarrow X$ is given by

$$
\left[x_{0}, \ldots, x_{r-1}\right] \mapsto\left[f_{d-1}\left(x_{0}, \ldots, x_{r-1}\right) x_{0}, \ldots, f_{d-1}\left(x_{0}, \ldots, x_{r-1}\right) x_{r-1},-f_{d}\left(x_{0}, \ldots, x_{r-1}\right)\right] .
$$

The map $\pi$ is called the stereographic projection of $X$ from $p$. Its indeterminacy locus is $p$. Each line through $p$ contained in $X$ gets contracted to a point under $\pi$. The set of all such lines is defined by the equations $\left\{f_{d}=f_{d-1}=0\right\}$. This is the indeterminacy locus of $\pi^{-1}$, whereas the hypersurface of $H$ with equation $\left\{x_{r}=f_{d-1}=0\right\}$ is contracted to $p$ by the map $\pi^{-1}$.

1.3. Monoidal extensions of rational maps. Let $\omega: \mathbb{P}^{r} \rightarrow-\mathbb{P}^{r}$ be a dominant rational map defined, in homogeneous coordinates, as in (1). The homogeneous polynomials $F_{0}, \ldots, F_{r}$ have the same degree $\delta>0$ and are coprime. We construct a monoidal extension $\Omega$ of $\omega$ as follows. First, we embed $\mathbb{P}^{r}$ in $\mathbb{P}^{r+1}$ as the hyperplane $H=\left\{x_{r+1}=0\right\}$ and we consider the point $p=[0, \ldots, 0,1] \in \mathbb{P}^{r+1}$. Fix an integer $d \geq \delta$, a nonzero polynomial $h\left(x_{0}, \ldots, x_{r}\right)$ of degree $d-\delta$ and an irreducible monoid of degree $d$ with vertex at $p$ defined by

$$
f\left(x_{0}, \ldots, x_{r+1}\right)=f_{d-1}\left(x_{0}, \ldots, x_{r}\right) x_{r+1}+f_{d}\left(x_{0}, \ldots, x_{r}\right)=0 .
$$

Then we let $\Omega: \mathbb{P}^{r+1} \rightarrow \mathbb{P}^{r+1}$ be defined by

$$
\left[x_{0}, \ldots, x_{r+1}\right] \mapsto\left[h\left(x_{0}, \ldots, x_{r}\right) F_{0}\left(x_{0}, \ldots, x_{r}\right), \ldots, h\left(x_{0}, \ldots, x_{r}\right) F_{r}\left(x_{0}, \ldots, x_{r}\right), f\left(x_{0}, \ldots, x_{r+1}\right)\right] .
$$

Note that $p$ is an indeterminacy point of $\Omega$. If $\pi: \mathbb{P}^{r+1} \rightarrow-\mathbb{P}^{r}$ is the projection from $p$ to $H$, we have

$$
\pi \circ \Omega=\omega \circ \pi \text {. }
$$


Lemma 1.1. The map $\Omega: \mathbb{P}^{r+1} \rightarrow \mathbb{P}^{r+1}$ is dominant and has the same degree as $\omega$. Hence $\Omega$ is a Cremona transformation if and only if $\omega$ is.

Proof. By definition, the degree of the map $\Omega$ coincides with the degree of the induced field extension. Let $y=\left[y_{0}, \ldots, y_{r+1}\right] \in \mathbb{P}^{r+1}$ be a general point and let $y^{\prime}=\left[y_{0}, \ldots, y_{r}\right]=\pi(y)$. The rational map $\Omega$ may be written as $\Omega(y)=\left[F_{0}\left(y^{\prime}\right): \ldots: F_{r}\left(y^{\prime}\right):\left(f_{d-1}\left(y^{\prime}\right) y_{r+1}+f_{d}\left(y^{\prime}\right)\right) / h\left(y^{\prime}\right)\right]$. In particular, $\Omega\left(\mathbb{C}\left(y_{0}, \ldots, y_{r}\right)\right)=$ $\omega\left(\mathbb{C}\left(y_{0}, \ldots, y_{r}\right)\right) \subset \mathbb{C}\left(y_{0}, \ldots, y_{r}\right)$, while $\Omega(y)_{r+1}$ is linear in $y_{r+1}$ over $\Omega\left(\mathbb{C}\left(y_{0}, \ldots, y_{r}\right)\right)$. The field extension has degree $\left[\mathbb{C}\left(y_{0}, \ldots, y_{r+1}\right): \Omega\left(\mathbb{C}\left(y_{0}, \ldots, y_{r}\right)\right)\right]=\left[\mathbb{C}\left(y_{0}, \ldots, y_{r}\right): \omega\left(\mathbb{C}\left(y_{0}, \ldots, y_{r}\right)\right)\right]$ and the lemma follows.

The indeterminacy locus of $\Omega$ (as a scheme) is the union of the cone over the locus of indeterminacy of $\omega$ with vertex $p$ intersected with the monoid $\{f=0\}$ and the codimension two subvariety $\{h=f=0\}$. The reduced fundamental locus of $\Omega$ is the union of the hypersurface $\{h=0\}$ and the cone over the fundamental locus of $\omega$ with vertex $p$.

1.4. Triangular Cremona transformations. Triangular Cremona transformations are obtained as iterated applications of monoidal extensions, as we now explain. Consider a rational map $\tau: \mathbb{P}^{r} \rightarrow \mathbb{P}^{r}$ defined, in affine coordinates over $\left\{x_{0} \neq 0\right\}$, by formulas of the type

$$
\left(x_{1}, \ldots, x_{r}\right) \rightarrow\left(f_{1}\left(x_{1}\right), \ldots, f_{i}\left(x_{1}, \ldots, x_{i}\right), \ldots f_{r}\left(x_{1}, \ldots, x_{r}\right)\right)
$$

where each $f_{i}\left(x_{1}, \ldots, x_{i}\right) \in K\left(x_{1}, \ldots, x_{i-1}\right)\left[x_{i}\right]$ is nonconstant and linear in $x_{i}$, for $1 \leqslant i \leqslant r$. If $f_{i}\left(x_{1}, \ldots, x_{i}\right) \in$ $K\left[x_{1}, \ldots, x_{i}\right]$ for all $1 \leqslant i \leqslant r$, the indeterminacy locus of $\tau$ is contained in the hyperplane at infinity $\left\{x_{0}=0\right\}$. Any such map $\tau$ is birational, with inverse of the same type. To find $\tau^{-1}$, we have to solve the system

$$
y_{i}=f_{i}\left(x_{1}, \ldots, x_{i}\right), \quad 1 \leqslant i \leqslant r
$$

in the indeterminates $x_{1}, \ldots, x_{r}$. This can be done stepwise as follows. From the linear expression $y_{1}=f_{1}\left(x_{1}\right)$ we find a linear polynomial $g_{1}$ such that $x_{1}=g_{1}\left(y_{1}\right)$. Given $i>1$, assume we know that

$$
x_{h}=g_{h}\left(y_{1}, \ldots, y_{h}\right), \quad \text { for } \quad 1 \leqslant h<i \leqslant n
$$

where $g_{h}\left(y_{1}, \ldots, y_{h}\right) \in K\left(y_{1}, \ldots, y_{h-1}\right)\left[y_{h}\right]$ is linear in $y_{h}$. From $y_{i}=f_{i}\left(x_{1}, \ldots, x_{i}\right)$ we obtain the expression $x_{i}=\xi\left(x_{1}, \ldots, x_{i-1}, y_{i}\right)$, where $\xi\left(x_{1}, \ldots, x_{i-1}, y_{i}\right) \in K\left(x_{1}, \ldots, x_{i-1}\right)\left[y_{i}\right]$ is linear in $y_{i}$. Substituting $x_{h}$ from (3), we conclude that $x_{i}=g_{i}\left(y_{1}, \ldots, y_{i}\right)$ with $g_{i}\left(y_{1}, \ldots, y_{i}\right) \in K\left(y_{1}, \ldots, y_{i-1}\right)\left[y_{i}\right]$ of degree 1 in $y_{i}$.

Example 1.2. Fix an integer $n \geq 2$ and use the same notation as above. The following Cremona quadratic transformation $\omega_{n}$ of $\mathbb{P}^{\left(\begin{array}{c}n+1 \\ 2\end{array}\right)}$ is triangular

$$
\left[x_{0}, \ldots, x_{i}, \ldots, x_{i j}, \ldots\right] \rightarrow\left[x_{0}^{2}, \ldots, x_{0} x_{i}, \ldots, x_{0} x_{i j}-x_{i} x_{j}, \ldots\right], \text { where } 1 \leq i<j \leq n .
$$

The inverse is

$$
\left[y_{0}, \ldots, y_{i}, \ldots, y_{i j}, \ldots\right] \rightarrow\left[y_{0}^{2}, \ldots, y_{0} y_{i}, \ldots, y_{0} y_{i j}+y_{i} y_{j}, \ldots\right]
$$

From a geometric viewpoint, $\omega_{n}$ is defined by a linear system $\mathcal{L}$ of quadrics as follows. Consider the coordinate hyperplane $\Pi=\left\{x_{0}=0\right\}$. Let $S$ be the linear subspace of $\Pi$ with equations $\left\{x_{0}=x_{1}=\ldots=x_{n}=0\right\}$ and let $S^{\prime}$ be the the complementary subspace in $\Pi$ with equations $\left\{x_{0}=x_{12}=\ldots=x_{i j}=\ldots=x_{(n-1) n}=\right.$ $0\}$, where $1 \leqslant i<j \leqslant n$. Then $\mathcal{L}$ cuts out the complete linear system of quadric cones on $\Pi$ that are singular along $S$ and that pass through the $n$ independent points $p_{i} \in S^{\prime}$, where $p_{i}$ is the torus-fixed point with all coordinates 0 but $x_{i}$, with $1 \leqslant i \leqslant n$. After splitting off $\Pi$ from $\mathcal{L}$, the residual system consists of all hyperplanes containing $S$.

\section{Cremona equivalence}

An irreducible variety $X \subset \mathbb{P}^{r}$ is Cremona linearizable (CL) if there is a linearizing Cremona transformation of $\mathbb{P}^{r}$ which maps $X$ birationally onto a linear subspace. It is a consequence of Theorem 2.5 (presented in [12]) that, if $X$ is rational of dimension $n \leqslant r-2$ in $\mathbb{P}^{r}$, then $X$ is CL. In this section we recall this theorem and present a slightly different proof. 
2.1. Monoids and Cremona transformations. Let $X \subset \mathbb{P}^{r}$ be a monoid of degree $d$. Let $p_{1}, p_{2} \in X$ be two vertices, let $H_{1}, H_{2}$ be hyperplanes with $p_{i} \notin H_{i}$, and consider the stereographic projections of $X$ from $p_{i}$, which is the restriction of the projection $\pi_{i}: \mathbb{P}^{r} \rightarrow H_{i}$ from $p_{i}$, with $i=1,2$. The map

$$
\pi_{X, p_{1}, p_{2}}:=\pi_{2} \circ \pi_{1}^{-1}: H_{1} \rightarrow H_{2}
$$

is a Cremona transformation. If $p_{1}=p_{2}=p$, then $\pi_{X, p}:=\pi_{X, p, p}$ does not depend on $X$, being the (linear) perspective of $H_{1}$ to $H_{2}$ with center $p$. From now on, we restrict to the case when $p_{1} \neq p_{2}$. In this setting, the map $\pi_{X, p_{1}, p_{2}}$ depends on $X$ and is in general nonlinear. In the following we assume that $H_{1}$ and $H_{2}$ have equations $x_{r}=0$ and $x_{r-1}=0$ respectively, and $p_{1}=[0, \ldots, 0,1], p_{2}=[0, \ldots, 0,1,0]$. The defining equation of $X$ has the form

$$
f_{d}\left(x_{0}, \ldots, x_{r-2}\right)+x_{r-1} g_{d-1}\left(x_{0}, \ldots, x_{r-2}\right)+x_{r} h_{d-1}\left(x_{0}, \ldots, x_{r-2}\right)+x_{r} x_{r-1} f_{d-2}\left(x_{0}, \ldots, x_{r-2}\right)=0 .
$$

Then

$$
\pi_{X, p_{1}, p_{2}}\left(\left[x_{0}, \ldots, x_{r-1}\right]\right)=\left[\left(f_{d-2} x_{r-1}+h_{d-1}\right) x_{0}, \ldots,\left(f_{d-2} x_{r-1}+h_{d-1}\right) x_{r-2},-f_{d}-x_{r-1} g_{d-1}\right] .
$$

Lemma 2.1. Let $Z \subset \mathbb{P}^{r}$, with $r \geqslant 3$, be an irreducible variety of dimension $r-2$ and let $p \in \mathbb{P}^{r}$ be such that the projection of $Z$ from $p$ is birational to its image. For $d \gg 0$ there is a monoid of degree $d$ with vertex $p$, containing $Z$ but not containing the cone $C_{p}(Z)$ over $Z$ with vertex $p$.

Proof. Let $V \rightarrow \mathbb{P}^{r}$ be the blow-up of $\mathbb{P}^{r}$ at $p$. We denote by $E$ the exceptional divisor and by $H$ the proper transform of a general hyperplane of $\mathbb{P}^{r}$ and by $Z^{\prime}$ the proper transform of $Z$.

Consider $M_{d}=|d H-(d-1) E|=|(d-1)(H-E)+H|$, i.e. the proper transform on $V$ of the linear system of monoids we are interested in. We have

$$
\operatorname{dim}\left(M_{d}\right)=\frac{2 d^{r-1}}{(r-1) !}+\frac{(r-1) d^{r-2}}{(r-2) !}+O\left(d^{r-3}\right) .
$$

Since the projection of $Z$ from $p$ is birational, the line bundle $\mathcal{O}_{Z^{\prime}}(H-E)$ is big and nef. Then by [10, Theorem 1.4.40, Vol. I, p. 69] it follows that

$$
h^{0}\left(Z^{\prime}, \mathcal{O}_{Z^{\prime}}(d(H-E))\right)=\frac{\delta}{(r-2) !} d^{r-2}+O\left(d^{r-3}\right), \text { for } d \gg 0
$$

where $\delta=(H-E)^{r-2} \cdot Z^{\prime}>0$ is the degree of the variety obtained under the projection of $Z$ from $p$, i.e. the degree of the cone $C_{p}(Z)$. Thus, if $M_{d}^{\prime}$ is the sublinear system of $M_{d}$ of the divisors containing $Z^{\prime}$, then

$$
\operatorname{dim}\left(M_{d}^{\prime}\right) \geqslant \operatorname{dim}\left(M_{d}\right)-h^{0}\left(Z^{\prime}, \mathcal{O}_{Z^{\prime}}(d(H-E))\right)=\frac{2 d^{r-1}}{(r-1) !}+\frac{(r-1-\delta) d^{r-2}}{(r-2) !}+O\left(d^{r-3}\right) .
$$

We let $M_{d}^{\prime \prime}$ be the sublinear system of $M_{d}^{\prime}$ of the divisors containing the proper transform $Y$ of the cone $C_{p}(Z)$, which is a hypersurface of degree $\delta$, i.e. $Y \in|\delta(H-E)|$. Hence, $M_{d}^{\prime \prime} \subseteq|(d-\delta-1)(H-E)+H|$ so by (4) we have

$$
\operatorname{dim}\left(M_{d}^{\prime \prime}\right) \leq \frac{2(d-\delta)^{r-1}}{(r-1) !}+\frac{(r-1)(d-\delta)^{r-2}}{(r-2) !}+O\left(d^{r-3}\right)=\frac{2 d^{r-1}}{(r-1) !}+\frac{(r-1-2 \delta) d^{r-2}}{(r-2) !}+O\left(d^{r-3}\right) .
$$

Hence

$$
\operatorname{dim}\left(M_{d}^{\prime}\right)-\operatorname{dim}\left(M_{d}^{\prime \prime}\right)=\frac{\delta d^{r-2}}{(r-2) !}+O\left(d^{r-3}\right)>0, \quad \text { for } \quad d \gg 0
$$

as we wanted to show.

Lemma 2.2. Let $Z \subset \mathbb{P}^{r}$ be an irreducible variety of positive dimension $n \leqslant r-3$ and let $p_{1}, p_{2} \in \mathbb{P}^{r}$ be distinct points such that the projection of $Z$ from the line $\ell$ joining $p_{1}$ and $p_{2}$ is birational to its image. For $d \gg 0$ there is a monoid of degree $d$ with vertices $p_{1}$ and $p_{2}$, containing $Z$ but not containing any of the cones $C_{i}(Z)$ over $Z$ with vertices $p_{i}$, for $i=1,2$.

Proof. We start with the following

Claim. It suffices to prove the assertion for $n=r-3$. 
Proof of the Claim. Consider the projection of $\mathbb{P}^{r}$ to $\mathbb{P}^{n+3}$ from a general linear subspace $\Pi$ of dimension $r-n-4$ and call $Z^{\prime}, p_{1}^{\prime}, p_{2}^{\prime}, \ell^{\prime}$ the projections of $Z, p_{1}, p_{2}, \ell$ respectively. Then $Z^{\prime}$ is birational to $Z$ and it is still true that the projection of $Z^{\prime}$ form $\ell^{\prime}$ is birational to its image. The dimension of $Z^{\prime}$ is $n-3$.

Assume the assertion holds for $Z^{\prime}, p_{1}^{\prime}, p_{2}^{\prime}$ and let $F^{\prime} \subset \mathbb{P}^{n+3}$ be a monoid of degree $d \gg 0$ with vertices $p_{1}^{\prime}, p_{2}^{\prime}$ containing $Z^{\prime}$ but not $C_{i}\left(Z^{\prime}\right)$, for $i=1,2$. Let $F \subset \mathbb{P}^{r}$ be the cone over $F^{\prime}$ with vertex $\Pi$. Then $F$ is a monoid with vertices $p_{1}, p_{2}$ containing $Z$. It does not contain either one of $C_{i}(Z)$, for $i=1,2$, otherwise $F^{\prime}$ would contain one of the cones $C_{i}\left(Z^{\prime}\right)$, for $i=1,2$, contradicting our hypothesis on $F^{\prime}$.

We can thus assume from now on that $n=r-3$. Fix two hyperplanes $H_{1}$ and $H_{2}$, where $p_{1} \notin H_{1}$ and $p_{2} \notin H_{2}$. Let $Z_{1}$ and $Z_{2}$ be the projections from $p_{1}$ and $p_{2}$ to $H_{1}$ and $H_{2}$, respectively. We set $p_{3-i}^{\prime}:=\pi_{i}\left(p_{3-i}\right)$, for $i=1,2$. Our result follows by Lemma 2.1 and the following claim:

Claim. It suffices to prove that for $d \gg 0$, there is a monoid of degree $d$ in $H_{i}$ with vertex $p_{i}^{\prime}$ containing $Z_{i}$ but not containing the cone $C\left(Z_{i}\right)$ over $Z_{i}$ with vertex $p_{i}^{\prime}$, for $i=1,2$.

Proof of the Claim. Let $F_{i}^{\prime} \subset H_{i}$ be such a monoid, and let $F_{i}$ be the cone over $F_{i}^{\prime}$ with vertex $p_{3-i}$, for $i=1,2$. Then $F_{i}$ is a monoid with vertex $p_{i}$ (by construction, we can take any point in the line joining $p_{i}$ and $p_{3-i}$ as its vertex). In addition, $F_{i}$ contains $Z$ but does not contain $C_{i}(Z)$ (same argument as in the proof of the previous Claim). Then the assertion of Lemma 2.1 holds for a general linear combination $F$ of $F_{1}$ and $F_{2}$.

Let $Z, p_{1}, p_{2}$ be as in Lemma 2.2. Fix a general monoid $X \supset Z$ with vertices in $p_{1}$ and $p_{2}$; by Lemma 2.2 $X$ does not contain the cones $C_{i}(Z)$. Let $H_{1}$ and $H_{2}$ be hyperplanes such that $p_{i} \notin H_{i}$, for $i=1,2$. Let $Z_{i}$ be the projection of $Z$ from $p_{i}$ to $H_{i}$, for $i=1,2$. Then the map $\varphi:=\pi_{X, p_{1}, p_{2}}: H_{1} \rightarrow H_{2}$ is birational and $Z_{1}$ is not contained in the indeterminacy locus of both $\varphi$ and $\varphi^{-1}$. Thus $\varphi$ induces a birational transformation $\phi: Z_{1} \rightarrow Z_{2}$. For future reference we summarize this construction in the following Proposition.

Proposition 2.3. In the above setting, the double projection $\varphi: H_{1} \rightarrow H_{2},\left(\right.$ resp. $\left.\varphi^{-1}\right)$ is defined at the general point of $Z_{1}$ (resp. of $Z_{2}$ ), hence it defines a birational map $\phi: Z_{1} \rightarrow Z_{2}$ (resp. $\phi^{-1}: Z_{2} \rightarrow Z_{1}$ ).

2.2. Cremona equivalence. Let $X, Y \subset \mathbb{P}^{r}$ be irreducible, projective varieties. We say that $X$ and $Y$ are Cremona equivalent $(\mathrm{CE})$ if there is a Cremona transformation $\omega: \mathbb{P}^{r} \rightarrow \rightarrow \mathbb{P}^{r}$ such that $\omega\left[\right.$ resp. $\omega^{-1}$ ] is defined at the general point of $X$ [resp. of $Y$ ] and such that $\omega$ maps $X$ to $Y$ [accordingly $\omega^{-1}$ maps $Y$ to $X$ ]. This is an equivalence relation among all irreducible subvarieties of $\mathbb{P}^{r}$.

The following result is due to Mella and Polastri [12, Theorem 1]. We present an alternative proof, close to the original ideas, but more computational in spirit.

Remark 2.4. We take the opportunity of correcting a mistake in the proof of [12, Theorem 1]. In the notation of [12, Theorem 1], let $T_{i}=\varphi_{\mathcal{H}_{i}}(X), Y_{i}=T_{i} \cap\left(x_{n+1}=0\right)$ and $Z$ the cone over $T_{i}$ with vertex $q_{1}$. Let $S$ be the monoid containing $Y_{i}$ and $W_{i}$ the projection of $T_{i}$ onto $S$. Then $X_{i+1}=\pi_{q_{2}}\left(W_{i}\right)$ and not the projection of a general hyperplane section of $Z$ as written in the published paper.

Theorem 2.5. Let $X, Y \subset \mathbb{P}^{r}$, with $r \geqslant 3$, be two irreducible varieties of positive dimension $n<r-1$. Then $X, Y$ are $C E$ if and only if they are birationally equivalent.

Proof. We prove the nontrivial implication.

Claim. We may assume that the projection of $Y$ from any coordinate subspace of dimension $m$ is birational to its image if $r>n+m+1$ and dominant to $\mathbb{P}^{r-m-1}$ if $r \leq n+m+1$.

Proof of the Claim. If we choose the $r+1$ torus-fixed points of $\mathbb{P}^{r}$ to be generic (which we can do after a generic change of coordinates), then each one of the coordinate subspaces of a given dimension $m$ (spanned by $m+1$ coordinate points) is generic in the corresponding Grassmannian, hence the assertion follows.

Let $Z$ be a smooth variety and let $\phi: Z \rightarrow X$ and $\psi: Z \rightarrow Y$ be birational maps. Passing to affine coordinates, we may assume that $\phi$ and $\psi$ are given by equations

$$
x_{j}=\phi_{j}(t) \text {, and } y_{j}=\psi_{j}(t) \text {, for } 1 \leqslant j \leqslant r,
$$

where $\phi_{j}, \psi_{j}$ are rational functions on $Z$ and $t$ varies in a suitable dense open subset of $Z$.

We prove the theorem by constructing a sequence of birational maps $\varphi_{i}: Z \rightarrow X_{i} \subset \mathbb{P}^{r}$, with $X_{i}$ projective varieties, for $0 \leqslant i \leqslant r$, such that: 
(a) $\varphi_{0}=\phi$ and $\varphi_{r}=\psi$, thus $X_{0}=X$ and $X_{r}=Y$;

(b) for $0 \leqslant i \leqslant r-1$, there is a Cremona transformation $\omega_{i}: \mathbb{P}^{r} \rightarrow \mathbb{P}^{r}$, such that $\omega_{i}\left(\right.$ resp. $\left.\omega_{i}^{-1}\right)$ is defined at the general point of $X_{i}$ (resp. of $X_{i+1}$ ), and it satisfies $\omega_{i}\left(X_{i}\right)=X_{i+1}$ (accordingly, $\left.\omega_{i}^{-1}\left(X_{i+1}\right)=X_{i}\right)$ and $\varphi_{i+1}=\omega_{i} \circ \varphi_{i}$

The construction is done recursively. We assume $\varphi_{i}$ is of the form

$$
\varphi_{i}(t)=\left(\tilde{\phi}_{i, 1}(t), \ldots, \tilde{\phi}_{i, r-i}(t), \psi_{r-i+1}(t), \ldots, \psi_{r}(t)\right), \text { for } t \in Z \text { and } 0 \leqslant i \leqslant r-1,
$$

where the $\tilde{\phi}_{i, j}$ 's are suitable rational functions on $Z$. For $i=0$, the starting case, we fix $\tilde{\phi}_{0, i}=\phi_{i}$ for all $0 \leqslant i \leqslant r$. Thus, requirement (a) is satisfied.

Assume $0 \leqslant i \leqslant r-1$. In order to perform the step from $i$ to $i+1$, we consider the map

$$
g_{i}: Z \rightarrow \mathbb{A}^{r+1}, \quad g_{i}(t)=\left(\tilde{\phi}_{i, 1}(t), \ldots, \tilde{\phi}_{i, r-i}(t), \psi_{r-i}(t), \psi_{r-i+1}(t), \ldots, \psi_{r}(t)\right) .
$$

Let $Z_{i}$ be the closure of the image of $g_{i}$, and $\pi_{j}: \mathbb{A}^{r+1} \rightarrow \mathbb{A}^{r}$ the projection to the coordinate hyperplane $\left\{x_{j}=0\right\}$ from the point at infinity of the axis $x_{j}$, for all $1 \leqslant j \leqslant r+1$. We have $\varphi_{i}=\pi_{r-i+1} \circ g_{i}$, and since $\varphi_{i}$ is birational onto its image, the same holds for $g_{i}$.

Claim. The projection of $Z_{i}$ from a general point of the space at infinity of the affine linear space $\Pi_{i}:=$ $\left\{x_{r-i+1}=\ldots=x_{r+1}=0\right\}$ is birational to its image.

Proof of the Claim. The variety $Z_{i}$ is not a hypersurface, then by [2, Theorem 1], the locus of points from which the projection is not birational has dimension strictly bounded by the dimension of $Z_{i}$. We may therefore assume that $\operatorname{dim}\left(\Pi_{i}\right)=r-i-1<n$. On the other hand the map $\psi$ is birational, therefore we may as well assume that $i<r-1$. So it remains to prove the result in the range $0<r-i-1<n$.

The projection of $Z_{i}$ from $\Pi_{i}$ is the closure of the image of the map

$$
h_{i}: Z \rightarrow \mathbb{A}^{i+1}, \quad h_{i}(t)=\left(\psi_{r-i}(t), \psi_{r-i+1}(t), \ldots, \psi_{r}(t)\right) .
$$

By the previous claim applied to $Z$, either $h_{i}$ is birational to its image (if $i \geqslant n$ ) or $h_{i}$ is dominant. In the former case the projection from a general point of $\Pi_{i}$ is also birational, so the assertion follows. In the latter case the cone over $Z_{i}$ with vertex $\Pi_{i}$ is the whole $\mathbb{P}^{r}$, and the assertion follows from [2, Theorem 1$]$.

By the Claim, we can make a general change of the first $r-i$ coordinates of $g_{i}$ so that $\varphi_{i+1}:=\pi_{r-i+i} \circ g_{i}$ is birational to its image $X_{i+1}$. Finally, iterated applications of Proposition 2.3 show that also requirement (b) is satisfied, thus ending the proof.

2.3. Linearizing Cremona. Theorem 2.5 ensures that any variety $X$ of dimension $n \leq r-2$ in $\mathbb{P}^{r}$ is $\mathrm{CE}$ to a hypersurface in a $\mathbb{P}^{n+1} \subset \mathbb{P}^{r}$. If, in addition, $X$ is rational, then it is CL, e.g. it is CE to the subspace $\left\{x_{n+1}=\ldots=x_{r}=0\right\}$. In particular, suppose that there is a linear subspace $\Pi$ of dimension $r-n-1$ of $\mathbb{P}^{r}$ such that the projection from $\Pi$ induces a birational map $\pi: X \rightarrow \mathbb{P}^{n}$. Equivalently, $X$ admits an affine parametrization of the form

$$
x_{i}=t_{i}, \quad \text { for } \quad 1 \leqslant i \leqslant n, \quad x_{j}=f_{j}\left(t_{1}, \ldots, t_{n}\right), \quad \text { for } \quad n+1 \leqslant j \leqslant r,
$$

where the $f_{i}$ 's are rational functions of $t_{1}, \ldots, t_{n}$. For instance, smooth toric varieties and Grassmannians enjoy this property (see Section 4).

Using (5), we define a Cremona map $\phi: \mathbb{P}^{r} \rightarrow \mathbb{P}^{r}$ in affine coordinates as

$$
\phi\left(x_{1}, \ldots, x_{r}\right)=\left(x_{1}, \ldots, x_{n}, x_{n+1}-f_{n+1}\left(x_{1}, \ldots, x_{n}\right), \ldots, x_{r}-f_{r}\left(x_{1}, \ldots, x_{n}\right)\right) .
$$

The map $\phi$ gives a birational equivalence between $X$ and the subspace $\left\{x_{n+1}=\ldots=x_{r}=0\right\}$, hence $\phi$ linearizes $X$. The above construction can be slightly modified to make it more general. Fix a collection of rational functions $g_{i}\left(x_{1}, \ldots, x_{i-1}\right)$ and $h_{i}\left(x_{1}, \ldots, x_{i-1}\right)$, with $n+1 \leqslant i \leqslant r$, with all the $h_{i}\left(x_{1}, \ldots, x_{i-1}\right)$ 's are nonzero. We replace the $i$-th coordinate of $\phi$ with the expression

$$
\phi_{i}\left(x_{1}, \ldots, x_{r}\right)=h_{i}\left(x_{1}, \ldots, x_{i-1}\right)\left(x_{i}-f_{i}\left(x_{1}, \ldots, x_{n}\right)\right)+g_{i}\left(x_{1}, \ldots, x_{i-1}\right), \quad i=n+1, \ldots, r .
$$

The following is clear:

Lemma 2.6. The image $\phi(X)$ is the linear subspace $\left\{x_{n+1}=\ldots=x_{r}=0\right\}$ if and only if the functions $g_{i}$ vanish on $X$ for $n+1 \leqslant i \leqslant r$. 


\section{SECANT AND TANGENTIAL VARIETIES}

In this section, we focus on Cremona transformations of secant and tangential varieties. Similar techniques can be applied to osculating varieties, although we will not do this here.

Definition 3.1. Let $X \subset \mathbb{P}^{r}$ be a variety of dimension $n$. The $k$-secant variety $\operatorname{Sec}_{k}(X)$ of $X(\operatorname{simply} \operatorname{Sec}(X)$ if $k=1)$ is the Zariski closure of the union of all $(k+1)$-secant linear spaces of dimension $k$ to $X$, i.e. those containing $k+1$ linearly independent points of $X$. The $k$-defect of $X$ is $\min \{r, n(k+1)+k\}-\operatorname{dim}\left(\operatorname{Sec}_{k}(X)\right)$ (which is nonnegative), and $X$ is $k$-defective if the $k$-defect is positive.

The $k$-secant variety of $X$ has expected dimension $n k+n+k$. It is parametrized as

$$
\psi: \operatorname{Sym}^{k+1}(X) \times \mathbb{P}^{k} \rightarrow \operatorname{Sec}_{k}(X) \subset \mathbb{P}^{r}, \quad\left(\left[p^{(0)}, \ldots, p^{(k)}\right],\left[s_{0}, \ldots, s_{k}\right]\right) \mapsto \sum_{j=0}^{k} s_{j} p^{(j)} .
$$

Assume that there is a codimension $n+1$ linear subspace $\Pi$ such that the projection from $\Pi$ induces a birational map $\pi: X \rightarrow \mathbb{P}^{n}$. From Section 2, we know that $X$ can be parametrized as in (5). Then, we can combine the maps $\psi$ and $\pi$ to simplify the parametrization of $\operatorname{Sec}_{k}(X)$, as we now show.

Pick affine variables $s_{1}, \ldots, s_{k}$ and set $s_{0}:=1-\sum_{j=1}^{k} s_{j}$ in (7). Consider $k+1$ vectors of unknowns

$$
\mathbf{t}_{i}=\left(t_{i 1}, \ldots, t_{i n}\right) \quad \text { for } \quad 0 \leqslant i \leqslant k .
$$

Then, $\operatorname{Sec}_{k}(X)$ is parametrized as follows

$$
x_{i}= \begin{cases}s_{0} t_{0 i}+s_{1} t_{1 i}+\ldots+s_{k} t_{k i} & \text { for } \quad 1 \leqslant i \leqslant n, \\ s_{0} f_{i}\left(\mathbf{t}_{0}\right)+s_{1} f_{i}\left(\mathbf{t}_{1}\right)+\ldots+s_{k} f_{i}\left(\mathbf{t}_{k}\right) & \text { for } \quad n+1 \leqslant i \leqslant r .\end{cases}
$$

We let $\phi$ be the Cremona transformation from (6), that linearizes $X$. Applying $\phi$ to $\operatorname{Sec}_{k}(X)$ gives

$$
x_{i}= \begin{cases}s_{0} t_{0 i}+s_{1} t_{1 i}+\ldots+s_{k} t_{k i} & \text { for } 1 \leqslant i \leqslant n, \\ s_{0} f_{i}\left(\mathbf{t}_{0}\right)+s_{1} f_{i}\left(\mathbf{t}_{1}\right)+\ldots+s_{k} f_{i}\left(\mathbf{t}_{k}\right)-f_{i}\left(s_{0} \mathbf{t}_{0}+s_{1} \mathbf{t}_{1}+\ldots+s_{k} \mathbf{t}_{k}\right) & \text { for } n+1 \leqslant i \leqslant r .\end{cases}
$$

This change of coordinates can be useful for computing geometric invariants of $X$, such as its $k$-defect. The next example, illustrates this situation.

Example 3.2. Suppose that the $f_{i}$ in (5) are quadratic polynomials. In this case, $X$ is a projection of the Veronese variety, hence it is 1 -defective. We write the homogeneous decomposition of $f_{i}$

$$
f_{i}=f_{i 0}+f_{i 1}+f_{i 2}, \quad \text { for } n+1 \leqslant i \leqslant r,
$$

where $f_{i j}$ is the homogeneous component of $f_{i}$ of degree $j$. Let $\Phi_{i}$ be the bilinear form associated to $f_{i 2}$. Then, the parametrization (8) yields the expression

$$
x_{i}= \begin{cases}s_{0} t_{0 i}+s_{1} t_{1 i}+\ldots+s_{k} t_{k i}, & \text { for } \quad 1 \leqslant i \leqslant n, \\ \sum_{j=0}^{k} s_{j}\left(1-s_{j}\right) f_{i 2}\left(\mathbf{t}_{j}\right)-2 \sum_{0 \leqslant u<v \leqslant k} s_{u} s_{v} \Phi_{i}\left(\mathbf{t}_{u}, \mathbf{t}_{v}\right), & \text { for } \quad n+1 \leqslant i \leqslant r .\end{cases}
$$

Suppose that $k=1$. Then

$$
x_{i}=s_{0}\left(1-s_{0}\right) f_{i 2}\left(\mathbf{t}_{0}\right)+s_{1}\left(1-s_{1}\right) f_{i 2}\left(\mathbf{t}_{1}\right)-2 s_{0} s_{1} \Phi_{i}\left(\mathbf{t}_{0}, \mathbf{t}_{1}\right), \text { for } n+1 \leqslant i \leqslant r .
$$

Since $s_{0}=1-s_{1}$, then $s_{0}\left(1-s_{0}\right)=s_{1}\left(1-s_{1}\right)=s_{0} s_{1}$ and we obtain

$$
x_{i}=s_{0} s_{1} f_{i 2}\left(\mathbf{t}_{1}-\mathbf{t}_{0}\right) \quad \text { for } \quad n+1 \leqslant i \leqslant r .
$$

Replacing $\mathbf{t}_{0}-\mathbf{t}_{1}$ with $\mathbf{u}:=\left(u_{1}, \ldots, u_{n}\right)$ and setting $\mathbf{t}_{0}=: \mathbf{t}=\left(t_{1}, \ldots, t_{n}\right)$ and $s_{1}=: s$ yields

$$
x_{i}= \begin{cases}t_{i}+s u_{i}, & \text { for } \quad 1 \leqslant i \leqslant n, \\ s(1-s) f_{i 2}(\mathbf{u}), & \text { for } \quad n+1 \leqslant i \leqslant r .\end{cases}
$$

The image of a general secant line is a conic with two points in the linear image of the variety $X$. The dimension of the secant variety can be deduced from the rank of the Jacobian of this parametrization. $\diamond$

Next we discuss the interplay between tangential varieties and Cremona transformations. 
Definition 3.3. Let $X \subset \mathbb{P}^{r}$ be a variety. The tangential variety $T(X)$ of $X$ is the Zariski closure of the union of all tangent spaces to $X$ at smooth points of $X$.

Assume that $X$ has dimension $n$. The tangential variety has expected dimension $2 n$. If $X$ is (locally) parametrized by a map

$$
\mathbf{t}=\left(t_{1}, \ldots, t_{n}\right) \in U \mapsto\left[x_{0}(\mathbf{t}), \ldots, x_{r}(\mathbf{t})\right] \in X,
$$

where $U \subset \mathbb{C}^{n}$ is a suitable nonempty open subset, then $T(X)$ is represented by

$$
\tau: U \times \mathbb{C}^{n} \rightarrow T(X), \quad(\mathbf{t}, \mathbf{s})=\left(t_{1}, \ldots, t_{n}, s_{1}, \ldots, s_{n}\right) \mapsto\left[x_{0}(\mathbf{t})+\sum_{j=1}^{n} s_{j} \frac{\partial x_{0}}{\partial t_{j}}(\mathbf{t}), \ldots, x_{r}(\mathbf{t})+\sum_{j=1}^{n} s_{j} \frac{\partial x_{r}}{\partial t_{j}}(\mathbf{t})\right] .
$$

Assume again that $X$ is described as in (5). Then, the parametric equations of $T(X)$ have a simplified expression

$$
x_{i}=\left\{\begin{array}{lll}
t_{i}+s_{i}, & \text { for } \quad 1 \leqslant i \leqslant n, \\
f_{i}(\mathbf{t})+\sum_{j=1}^{n} s_{j} \frac{\partial f_{i}}{\partial t_{j}}(\mathbf{t}), & \text { for } \quad n+1 \leqslant i \leqslant r .
\end{array}\right.
$$

Under the linearizing Cremona transformation $\phi$ from Section 2, the variety $T(X)$ has image

$$
x_{i}= \begin{cases}t_{i}+s_{i}, & \text { for } 1 \leqslant i \leqslant n, \\ f_{i}(\mathbf{t})-f_{i}(\mathbf{t}+\mathbf{s})+\sum_{j=1}^{n} s_{j} \frac{\partial f_{i}}{\partial t_{j}}(\mathbf{t}), & \text { for } n+1 \leqslant i \leqslant r .\end{cases}
$$

Example 3.4. Assume the $f_{i}$ 's in (5) are homogeneous quadratic polynomials. Then (9) becomes

$$
x_{i}= \begin{cases}t_{i}+s_{i}, & \text { for } \quad 1 \leqslant i \leqslant n, \\ -f_{i}(\mathbf{s}), & \text { for } \quad n+1 \leqslant i \leqslant r .\end{cases}
$$

Formula (10) describes a cone with vertex the space at infinity of the $n$-dimensional linear space $\left\{x_{n+1}=\right.$ $\left.\ldots=x_{r}=0\right\}$, over the variety parametrically represented by the last $n-r$ coordinates of (10)

$$
x_{i}=-f_{i}(\mathbf{s}), \quad \text { for } n+1 \leqslant i \leqslant r .
$$

In Section 4 we will see how the equations of secant and tangential varieties simplify in classical defective cases, as predicted by the above example. If the parametrization involves forms of degree higher than 2 , the tangent variety is in general no longer transformed to a cone. In Section 5 we will see alternative linearizing Cremonas that work better for certain varieties. For instance, for Segre varieties cumulant Cremonas enable us to write the tangential variety in the form (10) even though the parametrizing polynomials are not quadratic.

\section{Cremona linearization of some Classical Varieties}

Segre, Veronese and Grassmannian varieties and their secants play a key role in the study of determinantal varieties. Here we describe some triangular Cremona transformations that linearize these varieties, and we will compute the image of their secant varieties under these transformation. Similar considerations can be applied to Spinor varieties (see [1] for a parametrization of these varieties), and to Lagrangian Grassmannians $L G(n, 2 n)$, etc., on which we do not dwell here.

4.1. Segre varieties. The Segre variety $\operatorname{Seg}\left(r_{1}, \ldots, r_{k}\right)$ is the image of $\mathbb{P}^{r_{1}} \times \ldots \times \mathbb{P}^{r_{k}}$ under the Segre embedding in $\mathbb{P}^{r}$, with $r+1=\prod_{i=1}^{k}\left(r_{i}+1\right)$ (we may assume $r_{1} \geqslant r_{2} \geqslant \ldots \geqslant r_{k} \geqslant 1$ ). Sometimes we may use the exponential notation $\operatorname{Seg}\left(m_{1}^{h_{1}}, \ldots, m_{k}^{h_{k}}\right)$ if $m_{i}$ is repeated $h_{i}$ times, for $1 \leqslant i \leqslant k$.

In this section, we find Cremona linearizations for $\operatorname{Seg}(m, n)$ and we show how they simplify the equations for their secant varieties. In Section 5 we will extend this to higher Segre varieties.

We interpret $\mathbb{P}^{m n+m+n}$ as the space of nonzero $(m+1) \times(n+1)$ matrices modulo multiplication by a nonzero scalar, so we have coordinates $\left[x_{i j}\right]_{0 \leqslant i \leqslant n, 0 \leqslant j \leqslant m}$ in $\mathbb{P}^{m n+m+n}$. Then, $\operatorname{Seg}(m, n)$ is defined by the rank condition

$$
\operatorname{rk}\left(x_{i j}\right)_{0 \leqslant i \leqslant n, 0 \leqslant j \leqslant m}=1 .
$$


This condition amounts to equate to zero all $2 \times 2$ minors of the matrix $\mathbf{x}=\left(x_{i j}\right)_{0 \leqslant i \leqslant n, 0 \leqslant j \leqslant m}$. We pass to affine coordinates by setting $x_{00}=1$, and we let

$$
x=\left(\begin{array}{ccccc}
1 & x_{01} & x_{02} & \cdots & x_{0 n} \\
x_{10} & x_{11} & x_{12} & \cdots & x_{1 n} \\
\vdots & & & & \vdots \\
x_{m 0} & x_{m 1} & x_{m 2} & \cdots & x_{m n}
\end{array}\right)
$$

be the corresponding matrix. Then the affine equations of $\operatorname{Seg}(m, n)$ are $\left\{x_{i j}-x_{i 0} x_{0 j}=0\right\}_{1 \leqslant i \leqslant n, 1 \leqslant j \leqslant m}$. This shows that $\operatorname{Seg}(m, n)$ has parametric equations of type (5) with parameters $x_{i 0}, x_{0 j}$, for $1 \leqslant i \leqslant n, 1 \leqslant j \leqslant m$.

As in Section 2.3 a linearizing affine Cremona has equations (in vector form)

$$
\left(y_{i j}\right)_{0 \leq i \leq m, 0 \leq j \leq n,(i, j) \neq(0,0)}=\left(x_{i 0}, x_{0 j}, x_{i j}-x_{i 0} x_{0 j}\right)_{1 \leqslant i \leqslant n, 1 \leqslant j \leqslant m},
$$

which is of type $(2,2)$ and in homogeneous coordinates reads

$$
[\mathbf{y}]=\left[y_{i j}\right]_{0 \leq i \leq m, 0 \leq j \leq n)}=\left[x_{00}^{2}, x_{00} x_{i 0}, x_{00} x_{0 j}, x_{00} x_{i j}-x_{i 0} x_{0 j}\right]_{1 \leqslant i \leqslant n, 1 \leqslant j \leqslant m} .
$$

The indeterminacy locus has equations $\left\{x_{00}=x_{i 0} x_{0 j}=0\right\}_{1 \leqslant i \leqslant n, 1 \leqslant j \leqslant m}$ and the reduced fundamental locus $\left\{x_{00}=0\right\}$.

To see the image of the secant varieties, we perform column operations on $x$ and use (11) to see that

$$
\operatorname{rank}(x)=\operatorname{rank}\left(\begin{array}{ccccc}
1 & 0 & 0 & \cdots & 0 \\
y_{10} & y_{11} & y_{12} & \cdots & y_{1 n} \\
\vdots & & & & \vdots \\
y_{m 0} & y_{m 1} & y_{m 2} & \cdots & y_{m n}
\end{array}\right)=1+\operatorname{rank}\left(\begin{array}{cccc}
y_{11} & y_{12} & \cdots & y_{1 n} \\
\vdots & & & \vdots \\
y_{m 1} & y_{m 2} & \cdots & y_{m n}
\end{array}\right) .
$$

Therefore the $k$-secant variety to $\operatorname{Seg}(m, n)$ is mapped to the cone over the $(k-1)$-secant variety of $\operatorname{Seg}(m-$ $1, n-1)$ with vertex along the linear image of $\operatorname{Seg}(m, n)$.

Example 4.1. The (first) secant and tangent variety to $\operatorname{Seg}(2,2)$ is the cubic hypersurface defined by the $3 \times 3$-determinant

$$
\operatorname{det}(\mathbf{x})=x_{00}\left(x_{11} x_{22}-x_{12} x_{21}\right)-x_{01}\left(x_{10} x_{22}-x_{20} x_{12}\right)+x_{02}\left(x_{10} x_{21}-x_{20} x_{11}\right)=0 .
$$

In the new coordinates this hypersurface has the simpler binomial equation $y_{11} y_{22}-y_{12} y_{21}=0$.

4.2. Projectivized tangent bundles. The projectivized tangent bundle $T P^{n}$ over $\mathbb{P}^{n}$ is embedded in $\operatorname{Seg}(n, n)$ as the traceless nonzero $(n+1) \times(n+1)$-matrices modulo multiplication by nonzero scalar, i.e. as the hyperplane section $\operatorname{tr}(\mathbf{x})=0$ of $\operatorname{Seg}(n, n)$ in $\mathbb{P}^{n^{2}+2 n}$. On the affine chart $x_{00} \neq 0$, we view $T P^{n}$ as the set of rank 1 matrices of the form

$$
x=\left(\begin{array}{ccccc}
1 & x_{01} & x_{02} & \cdots & x_{0 n} \\
x_{10} & x_{11} & x_{12} & \cdots & x_{1 n} \\
\vdots & & & & \vdots \\
x_{n 0} & x_{n 1} & x_{n 2} & \cdots & -x_{11}-\ldots-x_{n-1, n-1}-1
\end{array}\right) .
$$

We can parametrize $T P^{n}$ with the $2 n-1$ coordinates $x_{0 i} \neq 0$, with $1 \leqslant i \leqslant n$, and $x_{i i}$, with $1 \leqslant i \leqslant n-1$. The parametric equations for the remaining coordinates are

$$
\begin{cases}x_{i 0}=\frac{x_{i i}}{x_{0 i}} & \text { for } 1 \leqslant i \leqslant n-1, \\ x_{n 0}=-\frac{1+x_{11}+\ldots+x_{n-1, n-1}}{x_{0 n}}, & \\ x_{i j}=\frac{x_{i i} x_{0 j}}{x_{0 i}}=x_{i 0} x_{0 j} & \text { for } 1 \leqslant i<j \leqslant n .\end{cases}
$$

According to Section 2.3 we have a linearizing Cremona map $\phi: \mathbb{P}^{n^{2}+2 n-1}-\rightarrow \mathbb{P}^{n^{2}+2 n-1}$ given in affine coordinates by

$$
\begin{array}{lr}
y_{0 i}=x_{0 i} & \text { if } 0 \leqslant i \leqslant n \\
y_{i i}=x_{i i} & \text { if } 1 \leqslant i \leqslant n-1 \\
y_{i 0}=x_{i i}-x_{i 0} x_{0 i} & \text { for } 1 \leqslant i \leqslant n-1 \\
y_{n 0}=-\left(1+x_{11}+\ldots+x_{n-1, n-1}+x_{n 0} x_{0 n}\right) & \\
y_{i j}=x_{i j}-x_{i 0} x_{0 j} & \text { for } 1 \leqslant i<j \leqslant n .
\end{array}
$$


Performing row operations on $x$ and using (12), we see that $x$ has rank $k$ if and only if

$$
y^{\prime}=\left(\begin{array}{ccccc}
y_{10} & y_{12} & y_{13} & \cdots & y_{1 n} \\
y_{21} & y_{20} & y_{23} & \cdots & y_{2 n} \\
\vdots & & & & \vdots \\
y_{n 1} & y_{n 2} & y_{n 3} & \cdots & y_{n 0}
\end{array}\right)
$$

has rank $k-1$. This shows that the $k$-th secant variety of $T P^{n}$ is mapped to a cone over the $(k-1)$-st secant variety of $\operatorname{Seg}(n-1, n-1)$ with vertex along the linear image of $T P^{n}$.

Example 4.2. The first secant variety of $T P^{2}$ coincides with the tangent variety and it is the cubic hypersurface defined in $\mathbb{P}^{7}$, with coordinates $\left[x_{i j}\right]_{0 \leqslant i \leqslant j \leqslant 2,(i, j) \neq(2,2)}$, by the equation

$$
\operatorname{det}(x)=x_{00}^{2} x_{11}+x_{00}\left(x_{11}^{2}+x_{12} x_{21}-x_{01} x_{10}\right)-x_{01}\left(x_{10} x_{11}+x_{20} x_{12}\right)-x_{02}\left(x_{10} x_{21}-x_{20} x_{11}\right)=0 .
$$

In the new coordinates it has the simpler equation $y_{12} y_{21}=y_{10} y_{20}$, which defines the cone over $\operatorname{Seg}(1,1)$ with vertex along the subspace $\left\{y_{12}=y_{21}=y_{10}=y_{20}=0\right\}$, the linear image of $T P^{2}$.

4.3. Veronese varieties. Consider the 2 -Veronese variety $V_{2, n}$ of quadrics in $\mathbb{P}^{n}$ embedded in $\mathbb{P}^{\frac{n(n+3)}{2}}$ with coordinates $\left[x_{i j}\right]_{0 \leqslant i \leqslant j \leqslant n}$. The following map $\phi$ is a linearizing affine $(2,2)$ Cremona transformation for $V_{2, n}$ defined on $\left\{x_{00} \neq 0\right\}$

$$
\left(x_{i j}\right)_{0 \leq i \leq j \leq n} \mapsto\left(y_{i j}\right)_{0 \leq i \leq j \leq n}=\left(x_{01}, \ldots, x_{0 n}, x_{i j}-x_{0 i} x_{0 j}\right)_{1 \leq i \leq j \leq n} .
$$

Its reduced fundamental locus is $\left\{x_{00}=0\right\}$ and the indeterminacy locus is $\left\{x_{00}=\ldots=x_{0 n}=0\right\}$.

We interpret $V_{2, n}$ as the set of rank 1 symmetric matrices $x=\left(x_{i j}\right)_{0 \leqslant i, j \leqslant n}$ with $x_{j i}=x_{i j}$ if $j<i$. The $(k-1)$-secant variety to $V_{2, n}$ is defined by the $k \times k$-minors of $x$. On $\left\{x_{00} \neq 0\right\}$ the rank of $x$ coincides with the rank of

$$
\left(\begin{array}{ccccc}
1 & 0 & 0 & \ldots & 0 \\
x_{01} & x_{11}-x_{01}^{2} & x_{12}-x_{01} x_{02} & \ldots & x_{1 n}-x_{01} x_{0 n} \\
\vdots & \vdots & \vdots & \vdots & \vdots \\
x_{0 n} & x_{1 n}-x_{01} x_{0 n} & x_{2 n}-x_{02} x_{0 n} & \ldots & x_{n n}-x_{0 n}^{2}
\end{array}\right)=\left(\begin{array}{ccccc}
1 & 0 & 0 & \ldots & 0 \\
x_{01} & y_{11} & y_{12} & \ldots & y_{1 n} \\
\vdots & \vdots & \vdots & \vdots & \vdots \\
x_{0 n} & y_{1 n} & y_{2 n} & \ldots & y_{n n}
\end{array}\right),
$$

So the $(k-1)$-secant variety to $V_{2, n}$ is mapped by $\phi$ to the cone over the $(k-2)$-secant variety of $V_{2, n-1}$ with vertex along the linear image of $V_{2, n}$ in $\mathbb{P}^{\frac{n(n+3)}{2}}$.

Example 4.3. The secant variety $\operatorname{Sec}\left(V_{2,2}\right)$ of the Veronese surface in $\mathbb{P}^{5}$ is mapped to the cone over the conic $V_{2,1}=\left\{y_{11} y_{22}-y_{12}^{2}=0\right\} \subset \mathbb{P}^{2}=\left\{y_{00}=y_{10}=y_{20}=0\right\}$ with vertex $\mathbb{P}^{2}=\left\{y_{11}=y_{12}=y_{22}=0\right\}$. $\diamond$

In general, the $d$-Veronese variety $V_{d, n}$ of $\mathbb{P}^{n}$ is embedded in $\mathbb{P}^{\left(\begin{array}{c}n+d \\ n\end{array}\right)-1}$ with coordinates $\left[x_{i_{0} \ldots i_{n}}\right]_{i_{0}+\ldots+i_{n}=d}$, with $i_{j} \geqslant 0$ for $0 \leqslant j \leqslant n$. Its projection from the linear space $\left\{x_{i_{0} \ldots i_{n}}=0\right\}_{i_{0} \geqslant d-1}$ to the $n$-space $\left\{x_{i_{0} \ldots i_{n}}=\right.$ $0\}_{i_{0}<d-1}$, is birational. Accordingly, we can find a Cremona linearizing map. We will treat the curve case in Section 4.6 but we will not dwell on the higher dimensional and higher degree cases.

4.4. Grassmannians of lines. In this section we present Cremona linearizations of Grassmannians of lines. Analogous linearizations exist for higher Grassmannians, an example of which we treat in Section 4.7.

Let $V$ be a complex vector space of dimension $n$. We can identify $V$ with $\mathbb{C}^{n}$, once we fix a basis $\left(\mathbf{e}_{0}, \ldots, \mathbf{e}_{n-1}\right)$ of $V$. The Plücker embedding maps the Grassmannian $G(2, n)$ of 2-dimensional vector subspaces (i.e. 2-planes) of $V$ into $\mathbb{P}^{\frac{n(n-1)}{2}}-1=\mathbb{P}\left(\wedge^{2} V\right)$, which we identify with the projective space associated to the vector space of antisymmetric matrices of order $n$, thus the coordinates are $\left[x_{i j}\right]_{0 \leq i<j \leq n-1}$.

Two vectors

$$
\xi_{0}=\left(\xi_{00}, \ldots, \xi_{0, n-1}\right), \quad \xi_{1}=\left(\xi_{10}, \ldots, \xi_{1, n-1}\right)
$$

in $V$ that span a 2-plane $W$ form the rows of a $2 \times n$-matrix $x$, whose minors are independent on the chosen points, up to a nonzero common factor. The Plücker point associated to $W$ is $\left[x_{i j}\right]_{0 \leq i<j \leq n-1}$, where $x_{i j}$ denotes the minor of $X$ obtained by choosing the $i$-th and $j$-th columns. 
The Plücker ideal $I_{2, n}$ is the homogeneous ideal of $G(2, n)$ in its Plücker embedding. This ideal is prime and it is generated by quadrics. More precisely, $I_{2, n}$ is generated by the $\left(\begin{array}{l}n \\ 4\end{array}\right)$ three terms Pücker relations

$$
x_{i j} x_{k l}-x_{i k} x_{j l}+x_{i l} x_{j k} \quad \text { for } 0 \leqslant i<j<k<l \leqslant n-1 .
$$

Using (13), in the open affine $\left\{x_{01} \neq 0\right\}$, we have parametric equations for $G(2, n)$ : the parameters are the $2 n-4$ coordinates $x_{i j}$ with $i=0,1$, and the equations for the remaining coordinates are

$$
x_{i j}=x_{0 i} x_{1 j}-x_{0 j} x_{1 i}, \text { for } 2 \leqslant i<j \leqslant n-1 .
$$

Hence $G(2, n)$ is rational, and a birational map $G(2, n) \rightarrow \mathbb{P}^{2 n-2}$ is given by projecting $G(2, n)$ from the linear span $\mathbb{P}^{\frac{n(n-5)}{2}}+2$ of $G(2, n-2)$ viewed inside $G(2, n)$ as the Grassmannian of 2-planes in $V^{\prime}=$ $\left\langle\mathbf{e}_{2}, \ldots, \mathbf{e}_{n-1}\right\rangle \subset V$.

According to Section 2.3, we have a triangular $(2,2)$-Cremona linearization $\varphi: \mathbb{P}^{\frac{n(n-1)}{2}-1} \rightarrow \mathbb{P}^{\frac{n(n-1)}{2}-1}$ of $G(2, n)$, given in affine coordinates by

$$
y_{i j}= \begin{cases}x_{i j} & \text { if } i=0,1,2 \leq j \leq n-1, \\ x_{i j}-x_{0 i} x_{1 j}+x_{0 j} x_{1 i} & \text { if } 2 \leqslant i<j \leqslant n-1 .\end{cases}
$$

The reduced fundamental locus is $\left\{x_{01}=0\right\}$, and the indeterminacy locus is the union of the two linear spaces $\left\{x_{01}=x_{02}=\ldots=x_{0(n-1)}=0\right\}$ and $\left\{x_{01}=x_{12}=\ldots=x_{1(n-1)}=0\right\}$.

On the complement of $\left\{x_{01}=0\right\}$, the Grassmannian $G(2, n)$ is the set of rank 2 matrices of the form

$$
x=\left(\begin{array}{cccccc}
0 & 1 & x_{02} & x_{03} & \ldots & x_{0 n} \\
-1 & 0 & x_{12} & x_{13} & \ldots & x_{1 n} \\
-x_{02} & -x_{12} & 0 & \ddots & \vdots & \vdots \\
-x_{03} & -x_{13} & \ddots & \ddots & \ddots & \vdots \\
\vdots & \vdots & \vdots & \ddots & \ddots & x_{n-2, n-1} \\
-x_{0, n-1} & -x_{1, n-1} & -x_{2, n-1} & \vdots & -x_{n-2, n-1} & 0
\end{array}\right) .
$$

Performing suitable column operations on $x$ and using the $y$-coordinates, we see that the rank of $x$ is 2 plus the rank of the matrix

$$
\left(\begin{array}{ccccc}
0 & y_{23} & \ldots & \ldots & y_{2, n-1} \\
\vdots & \vdots & \vdots & \vdots & \vdots \\
\vdots & \vdots & \vdots & 0 & y_{n-2, n-1} \\
-y_{2, n-1} & \ldots & \ldots & -y_{n-2, n-1} & 0
\end{array}\right) .
$$

Since $\operatorname{Sec}_{k}(G(2, n))$ is the set of antisymmetric matrices of rank $2 k+2$, we see that $\operatorname{Sec}_{k}(G(2, n))$ is mapped by $\varphi$ to the cone over $\operatorname{Sec}_{k-1}(G(2, n-2))$ with vertex along the linear image of $G(2, n)$.

Example 4.4. The first secant and tangent variety of $G(2,6)$ coincide and are defined by the Pfaffian cubic polynomial

$$
\begin{aligned}
& x_{01}\left(x_{23} x_{45}-x_{24} x_{35}+x_{25} x_{34}\right)-x_{02}\left(x_{13} x_{45}-x_{14} x_{35}+x_{15} x_{34}\right)+x_{03}\left(x_{12} x_{45}-x_{14} x_{25}+x_{15} x_{24}\right) \\
& -x_{04}\left(x_{12} x_{35}-x_{13} x_{25}+x_{15} x_{23}\right)+x_{05}\left(x_{14} x_{23}-x_{13} x_{24}+x_{12} x_{34}\right)=0 .
\end{aligned}
$$

In the $y$-coordinates, this hypersurface has a much simpler equation, namely the Plücker equation of $G(2,4)$

$$
y_{23} y_{45}-y_{24} y_{35}+y_{25} y_{34}=0 \text {. }
$$

Example 4.5. A different Cremona transformation that linearizes $G(2, n)$ was considered in [9], namely

$$
y_{0 i} \mapsto \frac{1}{x_{0 i}} \quad i=1, \ldots, n-1, \quad y_{i j} \mapsto \frac{x_{i j}}{x_{0 i} x_{0 j}} \quad 1 \leqslant i<j \leqslant n-1 .
$$

It maps $G(2, n)$ to the linear space defined by

$$
y_{i j}-y_{i k}+y_{j k}=0 \quad 1 \leqslant i<j<k \leqslant n-1 .
$$


This transformation is studied in [9] to compare various notions of convexity for lines.

4.5. Severi varieties. The Veronese surface $V_{2,2}$, the Segre variety $\operatorname{Seg}(2,2)$ and the Grassmannian $G(2,6)$ mentioned in Examples 4.3, 4.1 and 4.4 are Severi varieties, i.e. smooth 1 -defective varieties of dimension $n$ in $\mathbb{P}^{\frac{3}{2} n+2}$ (see [18]). There is one more Severi variety: the so-called Cartan variety of dimension 16 embedded in $\mathbb{P}^{26}$.

Let $X$ be a Severi variety. It is known that $X$ is swept out by a $n$-dimensional family $\mathcal{Q}$ of $\frac{n}{2}$-dimensional smooth quadrics, such that, given two distinct points $x, y \in X$, there is a unique quadric of $\mathcal{Q}$ containing $x, y$. If $Q \in \mathcal{Q}$, the projection of $X$ from the linear space $\langle Q\rangle$ of dimension $\frac{n}{2}+1$ to $\mathbb{P}^{n}$ is birational and, as usual by now, we get a Cremona linearization $\phi$ of $X$.

Being $X$ defective, its tangent and first secant varieties coincide. By Example 3.4 we see that $\phi$ maps $T(X)=\operatorname{Sec}(X)$ to the cone over $Q$ with vertex the $n$-dimensional linear image of $X$. This agrees with the contents of the previous examples and applies to the Cartan variety as well.

4.6. Rational normal curves. Let $V_{n}:=V_{1, n}$ be the rational normal curve of degree $n$ in $\mathbb{P}^{n}$

$$
V_{n}=\left\{\left[t^{n}, s t^{n-1}, \ldots, s^{n-1} t, s^{n}\right]:[s, t] \in \mathbb{P}^{1}\right\} \subset \mathbb{P}^{n}
$$

Let $\left[x_{0}, \ldots, x_{n}\right]$ be the coordinates of $\mathbb{P}^{n}$. Then $V_{n}$ is the determinantal variety

$$
\operatorname{rk}\left(\begin{array}{cccc}
x_{0} & x_{1} & \ldots & x_{n-1} \\
x_{1} & x_{2} & \ldots & x_{n}
\end{array}\right)=1 .
$$

Assume $n=2 k$ is even (similar considerations can be made in the odd case). Then, we can linearize $V_{n}$ via the following affine triangular $(2,2)$-Cremona map $\phi$ on $\left\{x_{0} \neq 0\right\}$

$$
y_{i}= \begin{cases}x_{1} & \text { if } i=1 \\ x_{i}-x_{i-1} x_{1} & \text { if } i>1 \text { and } i \text { is odd } \\ x_{i}-x_{\frac{i}{2}}^{2} & \text { otherwise. }\end{cases}
$$

The $\phi$-image of $V_{n}$ is the linear space $\left\{y_{2}=y_{3}=\ldots=y_{n}=0\right\}$. The reduced fundamental locus is $\left\{x_{0}=0\right\}$ and the indeterminacy locus is $\left\{x_{0}=x_{1}=\ldots=x_{k}=0\right\}$

The secant variety $\operatorname{Sec}\left(V_{n}\right)$ is defined by the $3 \times 3$-minors of the $3 \times(n-1)$ catalecticant matrix

$$
\left(\begin{array}{cccc}
x_{0} & x_{1} & \ldots & x_{n-2} \\
x_{1} & x_{2} & \ldots & x_{n-1} \\
x_{2} & x_{3} & \ldots & x_{n}
\end{array}\right),
$$

(see [5]), where we as usually set $x_{0}=1$. Using column operations, this matrix can be transformed into the following one expressed in terms of the $y$-coordinates

$$
\left(\begin{array}{cccc}
1 & 0 & \ldots & 0 \\
y_{1} & y_{2} & \ldots & y_{n-1} \\
y_{2}+y_{1}^{2} & y_{3} & \ldots & y_{n}
\end{array}\right) .
$$

This shows that $\operatorname{Sec}\left(V_{n}\right)$ is mapped by $\phi$ to the cone over a $V_{n-2}$ with vertex the line $\left\{y_{2}=\ldots=y_{n}=0\right\}$ which is the $\phi$-image of $V_{n}$. A similar situation occurs for all higher secant varieties of $V_{n}$. For instance, $\operatorname{Sec}_{k-1}\left(V_{n}\right)$ is the hypersurface defined by the catalecticant determinantal equation of degree $k+1=\frac{n}{2}+1$

$$
\operatorname{det}\left(\begin{array}{cccc}
x_{0} & x_{1} & \ldots & x_{k} \\
x_{1} & x_{2} & \ldots & x_{k+1} \\
\ldots & \ldots & \ldots & \ldots \\
x_{k} & x_{k+1} & \ldots & x_{n}
\end{array}\right)=0
$$

Hence, $\phi$ maps $\operatorname{Sec}_{k-1}\left(V_{n}\right)$ to the cone over $\operatorname{Sec}_{k-2}\left(V_{n-2}\right)$ with vertex the $\mathbb{P}^{1}$ obtained as the $\phi$-image of $V_{n}$. 
4.7. The Grassmannians $G(3,6)$. Let $X=\left(x_{i j}\right)_{1 \leqslant i, j \leqslant 3}$ and $Y=\left(y_{i j}\right)_{1 \leqslant i, j \leqslant 3}$ be $3 \times 3$-matrices, and let

$$
\left[x_{0}, X, Y, y_{0}\right]
$$

be coordinates in $\mathbb{P}^{19}$.

Let $A=\left(a_{i j}\right)_{1 \leqslant i, j \leqslant 3}$ be a $3 \times 3$ matrix. We denote by $A_{i j}$ the minor of $A$ obtained by deleting row $i$ and column $j$, so that

$$
\wedge^{2} A=\left(A_{i j}\right)_{1 \leqslant i, j \leqslant 3}, \text { and } \wedge^{3} A=\operatorname{det}(A) .
$$

We parametrize $G(3,6)$ as follows:

$$
\left(I_{3} \mid A\right) \in \mathbb{C}^{9} \mapsto\left(1, A, \wedge^{2} A, \wedge^{3} A\right) \in\left\{x_{0} \neq 0\right\} \subset \mathbb{P}^{19} .
$$

This parametrization is precisely the inverse of the birational projection of $G(3,6)$ from its tangent space at the point $[0,0,0,1]$.

By our discussion in Section 2.3, this gives rise to a family of triangular Cremona transformations linearizing $G(3,6)$

$$
\phi:\left[x_{0}, X, Y, y_{0}\right] \in \mathbb{P}^{19} \rightarrow\left[z_{0}, Z, W, w_{0}\right] \in \mathbb{P}^{19}
$$

where $Z=\left(z_{i j}\right)_{1 \leqslant i, j \leqslant 3}$ and $W=\left(w_{i j}\right)_{1 \leqslant i, j \leqslant 3}$.

We can view the determinant of $A$ in two ways: as a cubic polynomial in the entries of $A$ and as a bilinear quadric form in the variables $\left(a_{i j}, A_{i j}\right)$. This yields different Cremona transformations, one defined by quadrics whose inverse transformation is defined by cubics (a quadro-cubic transformation), the other defined by cubics with the inverse also defined by cubics (a cubo-cubic transformation).

Let us start with the quadro-cubic transformation $\phi$. On $\left\{x_{0} \neq 0\right\}$ it is defined by

$$
z_{i j}=x_{i j}, \quad w_{i j}=y_{i j}-X_{i j}, \quad w_{0}=y_{0}-\sum_{i=1}^{3}(-1)^{i+1} x_{1 i} y_{1 i} .
$$

The reduced fundamental locus is $\left\{x_{0}=0\right\}$ and the indeterminacy locus is $\left\{x_{0}=x_{i j}=0\right\}$. The inverse of $\phi$, on $\left\{z_{0} \neq 0\right\}$, is given by

$$
x_{i j}=z_{i j}, \quad y_{i j}=w_{i j}+Z_{i j}, \quad y_{0}=w_{0}+\sum_{i=1}^{3}(-1)^{i+1} z_{1 i}\left(w_{1 i}+Z_{1 i}\right) .
$$

The cubo-cubic Cremona transformation $\psi$ is given on the affine set $\left\{x_{0} \neq 0\right\}$ by the following expressions

$$
z_{i j}=x_{i j}, \quad w_{i j}=y_{i j}-X_{i j}, \quad w_{0}=y_{0}-\operatorname{det}(X) .
$$

Its reduced fundamental locus is $\left\{x_{0}=0\right\}$, and its indeterminacy locus is $\left\{x_{0}=x_{i j}=0\right\}$. The inverse Cremona transformation restricted to $\left\{z_{0} \neq 0\right\}$ is defined by

$$
x_{i j}=z_{i j}, \quad y_{i j}=w_{i j}+Z_{i j}, \quad y_{0}=w_{0}+\operatorname{det}(Z) .
$$

The image of $G(3,6)$ under both $\phi$ and $\psi$ is the linear space defined by $\left\{W=0, w_{0}=0\right\}$.

It is known that $\operatorname{Sec}(G(3,6))=\mathbb{P}^{19}$ (see $[6]$ ), while $T(G(3,6))$ is the quartic hypersurface defined by

$$
P=\left(x_{0} y_{0}-\operatorname{tr}(X Y)\right)^{2}+4 x_{0} \operatorname{det}(Y)+4 y_{0} \operatorname{det}(X)-4 \sum_{i, j} \operatorname{det}\left(X_{i j}\right) \operatorname{det}\left(Y_{j i}\right)=0,
$$

(see [14, p. 83]). We find the equation of $\phi(T(G(3,6)))$ by plugging (14) in $P$ (where $x_{0}=1$ ). We obtain a degree 6 equation

$$
z_{13}^{4} z_{22}^{2}-2 z_{12} z_{13}^{3} z_{22} z_{23}+\text { appr. } 600 \text { terms }=0 .
$$

Analogously, for $\psi(T(G(3,6)))$ we obtain

$$
z_{13}^{4} z_{22}^{2}-2 z_{12} z_{13}^{3} z_{22} z_{23}+\text { appr. } 600 \text { terms }=0 .
$$

Since $T(G(3,6))$ is singular along $G(3,6)$, the same happens for the above two sextics along $\left\{W=0, w_{0}=\right.$ $0\}$. In any event, none of these two linearizing Cremonas simplify the equation of $T(G(3,6))$, which actually becomes more complicated.

Similar considerations can be done for Grassmannians $G(n, 2 n)$ with $n \geq 4$. 


\section{Cumulant Cremonas}

As we saw in Section 4, there are several examples in which a Cremona linearization of a rational variety simplifies the equations of its secant varieties. Here is another instance of this behavior.

Example 5.1. Consider the Segre embedding $\Sigma_{n}$ of $\left(\mathbb{P}^{1}\right)^{n}$ in $\mathbb{P}^{2^{n}-1}$. In particular take the case $n=3$. Then, $\Sigma_{3}$ is parametrically given by the equations

$$
x_{1}=t_{1}, \quad x_{2}=t_{2}, \quad x_{3}=t_{3}, \quad x_{4}=t_{1} t_{2}, \quad x_{5}=t_{1} t_{3}, \quad x_{6}=t_{2} t_{3}, \quad x_{7}=t_{1} t_{2} t_{3} .
$$

We have $\operatorname{Sec}\left(\Sigma_{3}\right)=\mathbb{P}^{7}$, whereas $T\left(\Sigma_{3}\right)$ is a hypersurface of degree four in $\mathbb{P}^{7}$. Its defining equation is the so called hyperdeterminant (see [8]).

The linearizing Cremona transformation $\phi$ defined in (6) maps $\Sigma_{3}$ to the linear space $x_{4}=\ldots=x_{7}=0$. Following (9), the variety $T\left(\Sigma_{n}\right)$ is mapped under $\phi$ to a (symmetric) degree four hypersurface with defining equation

$x_{3}^{2} x_{4}^{2}+x_{2}^{2} x_{5}^{2}+x_{1}^{2} x_{6}^{2}+2\left(x_{1} x_{2} x_{5} x_{6}+x_{1} x_{3} x_{4} x_{6}+x_{2} x_{3} x_{4} x_{5}\right)+4 x_{4} x_{5} x_{6}-2 x_{7}\left(x_{1} x_{6}+x_{3} x_{4}+x_{2} x_{5}\right)+x_{7}^{2}=0$.

In this case the linearization process simplifies the equation of $T\left(\Sigma_{3}\right)$, but the degree remains the same. The question is: can we find a linearizing Cremona for $\Sigma_{3}$ which lowers the degree of $T\left(\Sigma_{3}\right)$ ? An affirmative answer to this question is given by cumulant Cremonas arising from algebraic statistics. Indeed, this family of Cremonas gives the following very simple equation for the image of $T\left(\Sigma_{3}\right)$ (see $[16,(2.1)]$ )

$$
x_{7}^{2}+4 x_{4} x_{5} x_{6}=0 .
$$

5.1. Binary cumulants. We recall the setting of binary cumulants from [16]. Let $\Pi(I)$ denote the set of all nonempty set partitions of $I \subseteq[n]:=\{1, \ldots, n\}$. We write $\pi=B_{1}|\cdots| B_{r}$ for a typical element of $\Pi(I)$, where all $\emptyset \neq B_{i} \subset I$ are the unordered disjoint blocks of $\pi$, with $I=\cup_{i=1}^{r} B_{i}$. For example, if $n=3$, then

$$
\Pi([3])=\{123,1|23,2| 13,3|12,1| 2 \mid 3\} .
$$

We denote by $|\pi|$ the number of blocks of $\pi \in \Pi(I)$.

Consider two copies of $\mathbb{P}^{2^{n}-1}=\mathbb{P}\left(\mathbb{C}^{2} \otimes \cdots \otimes \mathbb{C}^{2}\right)$ with coordinates $\left[x_{I}\right]_{I \subseteq[n]}$ and $\left[y_{I}\right]_{I \subseteq[n]}$. Following [16], we define the (binary) cumulant Cremona transformation or the (binary) cumulant change of coordinates

$$
\psi:\left[x_{I}\right]_{I \subseteq[n]} \in \mathbb{P}^{2^{n}-1} \rightarrow\left[y_{I}\right]_{I \subseteq[n]} \in \mathbb{P}^{2^{n}-1}
$$

via the formula

$$
y_{\emptyset}=x_{\emptyset}^{n}, \quad \text { and } \quad y_{I}=\sum_{\pi \in \Pi(I)}(-1)^{|\pi|-1}(|\pi|-1) ! x_{\emptyset}^{n-|\pi|} \prod_{B \in \pi} x_{B} \quad \text { for } \emptyset \neq I \subseteq[n],
$$

where the product in (15) is taken over all blocks $B$ of $\pi$ (we will call $\left[y_{I}\right]_{I \subseteq[n]}$ the cumulant coordinates). Note that $I$ is the maximal element in the poset $\Pi(I)$, hence $\psi$ is a triangular Cremona transformation. It linearizes $\Sigma_{n}$, which is mapped to the linear space $\left\{y_{I}=0\right\}_{|I| \geqslant 2}$ (see [16, Remark 3.4]), and $T\left(\Sigma_{n}\right)$ is toric in the cumulants coordinates (see [16, Theorem 4.1]).

The inverse map of $\psi$ is given by the standard Möbius inversion formula for the partition lattice $\Pi([n])$ (see [15, Proposition 3.7.1])

$$
x_{\emptyset}=y_{\emptyset}^{n}, \quad \text { and } \quad x_{I}=\sum_{\pi \in \Pi(I)} y_{\emptyset}^{n-|\pi|} \prod_{B \in \pi} y_{B} \quad \text { for } I \subseteq[n] .
$$

Both maps are morphisms on the open affine subsets $\left\{x_{\emptyset} \neq 0\right\}$ and $\left\{y_{\emptyset} \neq 0\right\}$, respectively.

Example 5.2. Fix $n=2$. Then

$$
y_{\emptyset}=x_{\emptyset}^{2}, y_{1}=x_{\emptyset} x_{1}, y_{2}=x_{\emptyset} x_{2}, \quad y_{12}=x_{\emptyset} x_{12}-x_{1} x_{2},
$$

which coincides with (11) in this case. The inverse is given by

$$
x_{\emptyset}=y_{\emptyset}^{2}, x_{1}=y_{\emptyset} y_{1}, x_{2}=y_{\emptyset} y_{2}, x_{12}=y_{\emptyset} y_{12}+y_{1} y_{2} .
$$

The fundamental locus is $\left\{x_{\emptyset}^{3}=0\right\}$.

Let $n=3$. Then

$$
\begin{aligned}
& y_{\emptyset}=x_{\emptyset}^{3}, \quad y_{i}=x_{\emptyset}^{2} x_{i}, \text { for } 1 \leqslant i \leqslant 3, \quad y_{i j}=x_{\emptyset}^{2} x_{i j}-x_{\emptyset} x_{i} x_{j}, \text { for } 1 \leq i<j \leq 3 \\
& y_{123}=x_{\emptyset}^{2} x_{123}-x_{\emptyset} x_{1} x_{23}-x_{\emptyset} x_{2} x_{13}-x_{\emptyset} x_{3} x_{12}+2 x_{1} x_{2} x_{3} .
\end{aligned}
$$


The inverse is

$$
\begin{aligned}
& x_{\emptyset}=y_{\emptyset}^{3}, x_{i}=y_{\emptyset}^{2} y_{i}, \text { for } 1 \leqslant i \leqslant 3, x_{i j}=y_{\emptyset}\left(y_{\emptyset} y_{i j}+y_{i} y_{j}\right), \text { for } 1 \leq i<j \leq 3 \\
& x_{123}=y_{\emptyset}^{2} y_{123}+y_{\emptyset}\left(y_{12} y_{3}+y_{13} y_{2}+y_{23} y_{1}\right)+y_{1} y_{2} y_{3} .
\end{aligned}
$$

The fundamental locus is now $\left\{x_{\emptyset}^{8}=0\right\}$.

5.2. Linearizing higher Segre varieties. The above construction can be generalized to $\operatorname{Seg}\left(r_{1}, \ldots, r_{k}\right) \subset$ $\mathbb{P}^{r}$ with $r+1=\prod_{i=1}^{3}\left(r_{i}+1\right)$, for any $k \geq 2$ and $r_{1} \geqslant \ldots \geqslant r_{k} \geqslant 1$. The case $k=2$ has been treated in Section 4.1. If $k=3$, let $\left[x_{i j k}\right]_{0 \leq i \leq r_{1}, 0 \leq j \leq r_{2}, 0 \leq k \leq r_{3}}$ be the coordinates on $\mathbb{P}^{r}$. Define a Cremona transformation by

$$
\begin{gathered}
y_{000}=x_{000}^{3}, \quad y_{i 00}=x_{000}^{2} x_{i 00}, \quad y_{0 j 0}=x_{000}^{2} x_{0 j 0}, \quad y_{00 k}=x_{000}^{2} x_{00 k}, \\
y_{i j 0}=x_{000}\left(x_{000} x_{i j 0}-x_{i 00} x_{0 j 0}\right), \quad y_{i 0 k}=x_{000}\left(x_{000} x_{i 0 k}-x_{i 00} x_{00 k}\right), \quad y_{0 j k}=x_{000}\left(x_{000} x_{0 j k}-x_{0 j 0} x_{00 k}\right), \\
y_{i j k}=x_{000}^{2} x_{i j k}-x_{000} x_{i 00} x_{0 j k}-x_{000} x_{0 j 0} x_{i 0 k}-x_{000} x_{00 k} x_{i j 0}+2 x_{i 00} x_{0 j 0} x_{00 k},
\end{gathered}
$$

where $i, j, k \geq 1$. This linearizes $\operatorname{Seg}\left(r_{1}, r_{2}, r_{3}\right)$ by mapping it to the linear space $\left\{y_{i j k}=0\right\}$ for all triples $(i, j, k) \in \prod_{i=1}^{3}\left\{0, \ldots, r_{i}\right\}$ with at least two nonzero coordinates.

This generalizes to any $k$ as follows (see [13, Sections 7 and 8]). Let $S(\mathbf{i}) \subseteq[n]$ be the support of $\mathbf{i}=$ $\left(i_{1}, \ldots, i_{k}\right) \in \prod_{i=1}^{k}\left\{0, \ldots, r_{i}\right\}$, i.e. the set of coordinates of nonzero entries in $\mathbf{i}$. For every $B \subseteq[k]$, we define the $k$-tuple $\mathbf{i}(B)$ that agrees with $\mathbf{i}$ on those indices in $B$ and is zero otherwise. We define the Cremona transformation $\psi: \mathbb{P}^{r} \rightarrow-\mathbb{P}^{r}$ by the formulas

$$
y_{\mathbf{i}}=\sum_{\pi \in \Pi(S(\mathbf{i}))}(-1)^{|\pi|-1}(|\pi|-1) ! x_{0 \cdots 0}^{n-|\pi|} \prod_{B \in \pi} x_{\mathbf{i}(B)}, \text { for all } \mathbf{i} \in \prod_{i=1}^{k}\left\{0, \ldots, k_{i}\right\} .
$$

The image of $\operatorname{Seg}\left(r_{1}, \ldots, r_{k}\right)$ lies in the subspace $\left\{y_{\mathbf{i}}=0\right\}_{|S(\mathbf{i})| \geqslant 2}$. This can be shown by mimicking the proof of Theorem 5.8 below, so we leave the proof to the reader.

5.3. $\mathcal{L}$-cumulant Cremonas. One of the advantages of working with cumulants is that the change of coordinates is conveniently encoded by the cumulant generating function [16]. However, to fully exploit the involved combinatorics, we will generalize cumulants to situations when such a generating function is not known. As we will see, $\mathcal{L}$-cumulants, introduced in [17], enjoy this property.

First we show how the homogeneous binary cumulant change of coordinates generalizes. We replace the partition lattice $\Pi(I)$ by a partial order set (poset) $\left(P,<_{P}\right)$ (or simply $(P,<)$ if there is no danger of confusion) with its associated Möbius function $\mu_{P}$. The function $\mu_{P}: P \times P \rightarrow \mathbb{Z}$ (or simply $\mu$ ) is recursively defined by $\mu(\pi, \pi)=1$ for all $\pi \in P, \mu(\pi, \nu)=0$ if $\pi \nless \nu$, and

$$
\mu(\pi, \nu)=-\sum_{\pi \leq \delta<\nu} \mu(\pi, \delta), \quad \text { for all } \pi<\nu \text { in } P .
$$

The two main features of this function that we will use in the rest of this section are the Möbius inversion formula and the product theorem, which we now recall. Even though they hold in a more general setting, we state them for finite posets, since this will suffice for our purposes.

Proposition 5.3 (Möbius inversion formula [15, Proposition 3.7.1]). Let $(P,<)$ be a finite poset and $f, g: P \rightarrow \mathbb{C}$. Then

$$
g(x)=\sum_{y \leq x} f(y) \quad \text { for all } x \in P \quad \text { if and only if } \quad f(x)=\sum_{y \leq x} g(y) \mu(y, x) \quad \text { for all } x \in P .
$$

Theorem 5.4 (Product theorem [15, Proposition 3.8.2]). Let $\left(P,<_{P}\right)$ and $\left(Q,<_{Q}\right)$ be finite posets and let $(P \times Q,<)$ be their product, with order given coordinatewise, i.e. $(p, q) \leq\left(p^{\prime}, q^{\prime}\right)$ if and only if $p \leq{ }_{P} p^{\prime}$ and $q \leq_{Q} q^{\prime}$. If $(p, q) \leq\left(p^{\prime}, q^{\prime}\right)$ in $P \times Q$, then

$$
\mu_{P \times Q}\left((p, q),\left(p^{\prime}, q^{\prime}\right)\right)=\mu_{P}\left(p, p^{\prime}\right) \mu_{Q}\left(q, q^{\prime}\right) .
$$

For further basic results concerning Möbius functions we refer the reader to [15, Chapter 3]. Some of them will be recalled later on in this section. 
The set partitions of a given nonempty set form a poset, where the order $<$ corresponds to refinement, that is, $\pi<\nu$ if $\pi$ refines $\nu$. To generalize cumulant Cremonas, we replace $\Pi([n])$ by a subposet $\mathcal{L}$ containing the maximal and minimal elements of $\Pi([n])$, i.e., the partitions

$$
\hat{0}=1|\ldots| n \text { and } \hat{1}=[n] .
$$

These two elements coincide if and only if $n=1$.

Let us fix such an $\mathcal{L}$. For each $I \subseteq[n]$, we construct a subposet $\mathcal{L}(I)$ of $\Pi(I)$ by restricting each partition in $\mathcal{L}$ to $I$. In particular, $\mathcal{L}([n])=\mathcal{L}$. Each poset $\mathcal{L}(I)$ has an associated Möbius function. To simplify notation, we denote all of them by $\mu$. Similarly, we denote the maximal and the minimal element of each poset by $\hat{0}$ and $\hat{1}$, so $\hat{1}=I$ in $\mathcal{L}(I)$. The identification will be clear from the context.

Given $\mathcal{L}$, we define a map $\psi_{\mathcal{L}}: \mathbb{P}^{2^{n}-1} \rightarrow \mathbb{P}^{2^{n}-1}$ as

$$
y_{I}= \begin{cases}\sum_{\pi \in \mathcal{L}(I)} \mu(\pi, \hat{1}) x_{\emptyset}^{n-|\pi|} \prod_{B \in \pi} x_{B} & \text { if } I \neq \emptyset, \\ x_{\emptyset}^{n} & \text { otherwise. }\end{cases}
$$

Here, $B \in \pi$ if it is a block of the partition. Note that

$$
y_{i}=x_{\emptyset}^{n-1} x_{i}, \text { for all } i \in[n] \text {, and, } y_{i j}=x_{\emptyset}^{n-2}\left(x_{\emptyset} x_{i j}-x_{i} x_{j}\right),
$$

do not depend on $\mathcal{L}$. Since $\hat{1} \in \mathcal{L}$, we know that $I$ is the maximal element of $\mathcal{L}(I)$ for every $I \subset[n]$. This implies that $\psi_{\mathcal{L}}$ is a triangular Cremona transformation. It is defined over the open set $\left\{x_{\emptyset} \neq 0\right\}$. We call such a map an $\mathcal{L}$-cumulant Cremona. Its fundamental locus is $\left\{x_{\emptyset}^{n^{2}-1}=0\right\}$.

Example 5.5. If $\mathcal{L}=\Pi([n])$, the Möbius function satisfies $\mu(\pi, \hat{1})=(-1)^{|\pi|-1}(|\pi|-1)$ !, so we recover the cumulant change of coordinates in (15).

To the other extreme, if $n>1$ and $\mathcal{L}=\{\hat{0}, \hat{1}\}$, then (16) becomes

$$
y_{I}= \begin{cases}x_{\emptyset}^{n-1} x_{I}-x_{\emptyset}^{n-|I|} \prod_{i \in I} x_{i} & \text { if } I \neq \emptyset, \\ x_{\emptyset}^{n} & \text { otherwise }\end{cases}
$$

which is the linearizing Cremona of $\Sigma_{n}$ arising, as in (6), from the affine parametrization of $\Sigma_{n}$ given by

$$
x_{I}=\prod_{i \in I} t_{i} \text {, for } \emptyset \neq I \subseteq[n] \text { and }\left(t_{1}, \ldots, t_{n}\right) \in \mathbb{C}^{n} .
$$

Example 5.6 (Interval partitions of $[n]$ ). Fix a positive integer $n$ and let $\mathcal{L}$ be the set of interval partitions of $[n]$, ordered by refinement. An interval partition of $[n]$ is obtained by cutting the sequence $1,2, \ldots, n$ into subsequences. For example, there are four interval partitions on [3], i.e., 123, $1|23,12| 3$ and $1|2| 3$.

The interval partitions form a poset isomorphic to the Boolean lattice of a set of $n-1$ elements. In particular, its Möbius function satisfies $\mu(\pi, \hat{1})=(-1)^{|\pi|-1}$ (c.f. [15, Example 3.8.3]). If $n=3$, this gives the following formulas for the map $\psi_{\mathcal{L}}$ from (16)

$$
\begin{gathered}
y_{\emptyset}=x_{\emptyset}^{3}, \quad y_{i}=x_{\emptyset}^{2} x_{i}(i=1,2,3), \quad y_{12}=x_{\emptyset}^{2} x_{12}-x_{\emptyset} x_{1} x_{2}, \quad y_{13}=x_{\emptyset}^{2} x_{13}-x_{\emptyset} x_{1} x_{3}, \quad y_{23}=x_{\emptyset}^{2} x_{23}-x_{\emptyset} x_{2} x_{3}, \\
y_{123}=x_{\emptyset}^{2} x_{123}-x_{\emptyset} x_{1} x_{23}-x_{\emptyset} x_{3} x_{12}+x_{1} x_{2} x_{3} .
\end{gathered}
$$

The formula for the inverse of $\psi_{\mathcal{L}}$ over $\left\{y_{\emptyset} \neq 0\right\}$ follows by the standard Möbius inversion formula on each poset $\mathcal{L}(I)$. Let us show how to do this. For every $\pi \in \Pi(I)$ we set

$$
x_{\pi}:=\prod_{B \in \pi} x_{B} .
$$

Given $I \subseteq[n]$ and $\nu \in \mathcal{L}(I)$, we define

$$
y_{\nu}:=\sum_{\substack{\pi \leq \nu \\ \pi \in \mathcal{L}(I)}} \mu(\pi, \nu) x_{\pi} .
$$

By the Möbius inversion formula on $\mathcal{L}(I)$, we conclude that

$$
x_{\nu}=\sum_{\substack{\pi \leq \nu \\ \pi \in \mathcal{L}(I)}} y_{\pi} \quad \text { for all } I \subset[n] \text { and } \nu \in \mathcal{L}(I) .
$$


In particular

$$
x_{I}=\sum_{\pi \in \mathcal{L}(I)} y_{\pi}, \text { for all } I \subset[n] .
$$

The following lemma ensures that, for each $\nu \in \mathcal{L}(I), y_{\nu}$ is a polynomial in the variables $y_{J}$ 's with $J \subseteq I$. It also show that $(20)$ and yields an explicit formula for $\psi_{\mathcal{L}}^{-1}$.

Lemma 5.7. For each $I \subset[n]$ and each $\nu \in \mathcal{L}(I)$, the variable $y_{\nu}$ is a polynomial in $y_{J}$ 's where $J$ runs over all subsets of each one of the blocks of $\nu$.

Proof. We prove the result by induction on the subsets of $[n]$. If $I=\emptyset$, there is nothing to prove since $y_{\emptyset}=1$. Suppose that $I \supsetneq \emptyset$ and that the result holds for all $J \subsetneq I$. If $\nu$ is a one block partition, there is nothing to prove. Assume that $\nu$ contains more than one block. By (17), (18) and (19) we obtain

$$
y_{\nu}=\sum_{\pi \leq \nu} \mu(\pi, \nu) \prod_{B \in \pi}\left(\sum_{\tau \in \mathcal{L}(B)} y_{\tau}\right) .
$$

Since all B's on the right-hand side are strictly included in $I$, the result follows by induction.

As it happens with the homogeneous cumulant change of coordinates (15), the map $\psi_{\mathcal{L}}$ linearizes $\Sigma_{n}$ :

Theorem 5.8. For any choice of $\mathcal{L}$, the map $\psi_{\mathcal{L}}$ from (16) linearizes $\Sigma_{n}$. Its image is the linear space $\Pi:=\left\{y_{I}=0\right\}_{I \subseteq[n],|I| \geq 2}$.

Proof. Denote by $a_{i}=\left[a_{i 0}, a_{i 1}\right]$ the coordinates of the $i$-th copy of $\mathbb{P}^{1}$ in $\left(\mathbb{P}^{1}\right)^{n}$. The Segre embedding $\sigma_{n}:\left(\mathbb{P}^{1}\right)^{n} \rightarrow \mathbb{P}^{2^{n}-1}$, maps $a=\left(a_{1}, \ldots, a_{n}\right)$ to the point in $\mathbb{P}^{2^{n}-1}$ whose $I$-th coordinate is

$$
a_{I}=\prod_{i \in I} a_{i 1} \prod_{i \notin I} a_{i 0}, \text { for every } I \subseteq[n] .
$$

We compute $\psi_{\mathcal{L}} \circ \sigma_{n}$ using (16). For every $I \subseteq[n]$ and every partition $\pi \in \mathcal{L}(I)$ we have

$$
a_{\emptyset}^{n-|\pi|} \prod_{B \in \pi} a_{B}=\prod_{i \in I}\left(a_{i 0}^{n-1} a_{i 1}\right) \prod_{i \notin I} a_{i 0}^{n}
$$

which does not depend on $\pi$. Therefore, the $I$-th coordinate of $\psi_{\mathcal{L}}\left(\sigma_{n}(a)\right)$ is

$$
b_{I}=\left(\prod_{i \notin I} a_{i 0}^{n} \prod_{i \in I}\left(a_{i 0}^{n-1} a_{i 1}\right)\right) \sum_{\pi \in \mathcal{L}(I)} \mu(\pi, \hat{1}) .
$$

If $|\mathcal{L}(I)| \geq 2$, Lemma 5.9 below, applied to $P=\mathcal{L}(I)$, yields $\sum_{\pi \in \mathcal{L}(I)} \mu(\pi, \hat{1})=0$. Combining this fact with (21), we conclude that the image of $\Sigma_{n}$ via $\psi_{\mathcal{L}}$ is contained in the linear space $\left\{y_{I}=0\right\}_{|\mathcal{L}(I)| \geq 2}$. Note that since $\hat{1}$ and $\hat{0}$ lie in $\mathcal{L}$, the condition $|\mathcal{L}(I)| \geq 2$ is equivalent to $|I| \geq 2$. So this linear space is $\Pi$, and it has dimension $n$. Moreover $\Sigma_{n}$ is not contained in the fundamental locus of $\psi_{\mathcal{L}}$, so the induced map $\psi_{\mathcal{L} \mid \Sigma_{n}}: \Sigma_{n} \rightarrow \Pi$ is birational.

Lemma 5.9. Let $(P, \leq)$ be a finite poset of size at least two with unique maximal and minimal elements $\hat{1}, \hat{0}$. Let $\mu$ be its Möbius function. Then,

$$
\sum_{x \in P} \mu(x, \hat{1})=0
$$

Proof. Consider the dual poset $\left(P^{*}, \leq^{*}\right)$ obtained by reversing the order in $(P, \leq)$. In particular, the roles of the minimal and maximal elements are exchanged, namely $\hat{0}^{*}=\hat{1}$ and $\hat{1}^{*}=\hat{0}$. The Möbius function $\mu^{*}$ of $P^{*}$ satisfies $\mu^{*}(x, y)=\mu(y, x)$ for all $(x, y) \in P \times P$ (see [15, page 120]). Therefore

$$
\sum_{x \in P} \mu(x, \hat{1})=\mu(\hat{0}, \hat{1})+\sum_{\hat{0}<x \leq \hat{1}} \mu(x, \hat{1})=\mu^{*}\left(\hat{0}^{*}, \hat{1}^{*}\right)+\sum_{\hat{0}^{*} \leq * x<^{*} \hat{1}^{*}} \mu^{*}\left(\hat{0}^{*}, x\right)=0,
$$

where the last equality follows from the recursive definition of $\mu^{*}$. 
5.4. Secant cumulants. As we mentioned earlier, one of the useful features of binary cumulants is that the tangential variety of $\Sigma_{n}$, expressed in cumulants, becomes toric. This is not the case, in general, for $\mathcal{L}$-cumulant Cremonas. However, with a careful choice of the defining poset $\mathcal{L}$ one may obtain other desired properties. For example, if $\mathcal{L}$ is the poset of interval partitions of [n] defined in Example 5.6, the Cremona transformation $\psi_{\mathcal{L}}$ is an involution.

The next example is related to $\operatorname{Sec}\left(\Sigma_{n}\right)$ (see [13], [17, Section 3.3]).

Example 5.10. In what follows, we parametrize the secant variety $\operatorname{Sec}\left(\Sigma_{n}\right)$ inside $\mathbb{P}^{2^{n}-1}$ starting from the parametrization of $\Sigma_{n}$ :

$$
p_{I}^{(0)}=\prod_{i \in[n] \backslash I} a_{i 0} \prod_{i \in I} a_{i 1}, \quad p_{I}^{(1)}=\prod_{i \in[n] \backslash I} b_{i 0} \prod_{i \in I} b_{i 1} \quad \text { for all } I \subseteq[n] .
$$

Denote by $\mathbf{A}$ the affine subspace given by $x_{\emptyset}=1$. The affine variety $\operatorname{Sec}\left(\Sigma_{n}\right) \cap \mathbf{A}$ is parametrized by

$$
x_{I}=\left(1-s_{1}\right) \prod_{i \in I} a_{i 1}+s_{1} \prod_{i \in I} b_{i 1} .
$$

Consider a sequence of two $\mathcal{L}$-cumulant transformations. The first one corresponds to the lattice $\mathcal{L}_{1}$ of all one-cluster partitions of $[n]$, i.e. partitions with at most one block of size greater than one. The second one comes from the lattice $\mathcal{L}_{2}$ of interval partitions of $[n]$. The first map $\psi_{1}: \mathbf{A} \rightarrow \mathbf{A}$ is defined by

$$
y_{I}=\sum_{A \subseteq I}(-1)^{|I \backslash A|} x_{A} \prod_{i \in I \backslash A} x_{i}, \quad \text { for } I \subseteq[n] .
$$

The second map $\psi_{2}: \mathbf{A} \rightarrow \mathbf{A}$ is given by 16, i.e.

$$
z_{I}=\sum_{\pi \in \mathcal{I}(I)}(-1)^{|\pi|-1} \prod_{B \in \pi} y_{B}, \quad \text { for } I \subseteq[n] .
$$

To see how this sequence of maps can be written as a single $\mathcal{L}$-cumulant transformation we refer to [17]. By [13, Lemma 3.1], for every $I \subseteq[n]$ such that $|I| \geq 2$, the result of $\psi_{2} \circ \psi_{1}$ applied to $\operatorname{Sec}\left(\Sigma_{n}\right)$ is

$$
z_{I}=s_{1}\left(1-s_{1}\right)\left(1-2 s_{1}\right)^{|I|-2} \prod_{i \in I}\left(b_{i 1}-a_{i 1}\right) .
$$

Taking $d_{i}=\left(1-2 s_{1}\right)\left(b_{i 1}-a_{i 1}\right)$ for $i \in[n]$, and $t=s_{1}\left(1-s_{1}\right)\left(1-2 s_{1}\right)^{-2}$ in (22) we conclude that that secant variety, when expressed in cumulants, becomes locally toric with $z_{I}=t \prod_{i \in I} d_{i}$ for $|I| \geq 2$.

This simple local description of $\operatorname{Sec}\left(\Sigma_{n}\right)$ can be generalized to the secant variety of the Segre product of projective spaces of arbitrary dimensions. This gives the following result:

Theorem 5.11 (see [13]). The secant variety $\operatorname{Sec}\left(\operatorname{Seg}\left(r_{1}, \ldots, r_{k}\right)\right)$ is covered by normal affine toric varieties. In particular, it has rational singularities.

It turns out that similar techniques can be applied to study the tangential variety $T\left(\operatorname{Seg}\left(r_{1}, \ldots, r_{k}\right)\right.$ ) (see [13] for details).

Acknowledgments: We thank Mateusz Michałek for his helpful comments on an early version of the paper. M. A. Cueto was partially supported by an AXA Mittag-Leffler postdoctoral fellowship (Sweden), by an Alexander von Humboldt Postdoctoral Research Fellowship and by an NSF postdoctoral fellowship DMS1103857 (USA). C. Ciliberto and M. Mella have been partially supported by the Progetto PRIN "Geometria sulle varietà algebriche" MIUR. P. Zwiernik was partially supported by an AXA Mittag-Leffler postdoctoral fellowship (Sweden), by Jan Draisma's Vidi grant from the Netherlands Organisation for Scientific Research (NWO) and by the European Union Seventh Framework Programme (FP7/2007-2013) under grant agreement PIOF-GA-2011-300975. This project started at the Institut Mittag-Leffler during the Spring 2011 program on "Algebraic Geometry with a View Towards Applications." We thank IML for its wonderful hospitality. 


\section{REFERENCES}

[1] E. Angelini, Higher secants of Spinor varieties. Boll. Unione Mat. Ital. (9) 4, no. 2, 213-235, 2011.

[2] A. Calabri and C. Ciliberto, On special projections of varieties: epitome to a theorem of Beniamino Segre, Adv. Geom. 1, 97-106, 2001.

[3] S. Cantat, The Cremona group in two variables, Proceedings of the sixth European Congress of Mathematics, 211-225, Europ. Math. Soc., 2013.

[4] B. Costa and A. Simis, New Constructions of Cremona Maps, Math. Res. Lett. 20(4), 629-645, 2013.

[5] I. Dolgachev, Classical Algebraic Geometry: A Modern View, Cambridge Univ. Press, 2012.

[6] R. Donagi, On the geometry of Grassmannians. Duke Math. Journal 44, 795-837, 1977.

[7] S. DRUEL, Existence des modèles minimaux pour les variétés de type général, Séminaire BOURBAKI, Novembre 2007, 2007-2008, no. 982.

[8] I. M. Gel'FAnd, M. M. Kapranov and A. V. Zelevinsky, Discriminants, resultants, and multidimensional determinants. Mathematics: Theory \& Applications. Birkhäuser Boston Inc., Boston, MA, 1994.

[9] J. E. Goodman, A. Holmsen, R. Pollack, K. Ranestad and F. Sottile, Cremona Convexity, Frame Convexity, and a Theorem of Santaló. Adv. Geom. 6, 301-321, 2006.

[10] R. Lazarsfeld, Positivity in Algebraic geometry, vol. I and II, Springer Verlag, 2004.

[11] L. Manivel And M. MichaŁeK, Secant varieties of minuscule and cominuscule orbits, arXiv:1401.1956v1, 9 Jan 2014.

[12] M. Mella And E. Polastri, Equivalent birational embeddings. Bull. Lond. Math. Soc. 41(1), 89-93, 2009.

[13] M. MichaŁek, L. Oeding and P. Zwiernik, Secant cumulants and toric geometry. arxiv:1212.1515v1, to appear on Int. Math. Res. Not.

[14] M. SAto and T. Kimura, A classification of irreducible prehomogeneous vector spaces and their relative invariants. Nagoya Math. J. 65, 1-155, 1977.

[15] R. P. Stanley, Enumerative combinatorics. Volume I. Cambridge Studies in Advanced Mathematics 49, Cambridge University Press, 2002.

[16] B. Sturmfels and P. Zwiernik, Binary cumulant varieties. Ann. Comb. 17, no. 1, 229-250 (2013).

[17] P. Zwiernik, L-cumulants, L-cumulant embeddings and algebraic statistics. J. Alg. Stat. 3, 11-43, 2012.

[18] F. L. Zak, Tangents and secants of algebraic varieties, Translations of Mathematical Monographs 127, Amer. Math. Soc., Providence, RI, 1993.

Università degli Studi di Roma Tor Vergata, Via della Ricerca Scientifica 1, 00133, Rome, Italy.

E-mail address: cilibert@axp.mat.uniroma2.it

Mathematics Department, Columbia University, MC 4403, 2990 Broadway, New York, Ny 10027, USA

E-mail address: macueto@math.columbia.edu

Dipartimento di Matematica e Informatica, Università di Ferrara, Via Machiavelli 35, 44100 Ferrara, Italia

E-mail address: mll@unife.it

University of Oslo, PB 1053 Blindern, 0316, Oslo, Norway

E-mail address: ranestad@math.uio.no

University of California Berkeley, Department of Statistics, CA 94720-3860, Berkeley, USA

E-mail address: pzwiernik@berkeley.edu 